Article

\title{
Specific Deletion of the FHA Domain Containing SLMAP3 Isoform in Postnatal Myocardium Has No Impact on Structure or Function
}

\author{
Taha Rehmani ${ }^{1}{ }^{\circledR}$, Jana Mlynarova ${ }^{1}$, Joseph Byers ${ }^{2}$, Maysoon Salih ${ }^{1}$ and Balwant S. Tuana ${ }^{1, *}$ \\ 1 Department of Cellular and Molecular Medicine, Faculty of Medicine, University of Ottawa, 451 Smyth Road, \\ Ottawa, ON K1H 8M5, Canada; trehmani@uottawa.ca (T.R.); jnmlynarova@gmail.com (J.M.); \\ msalih@uottawa.ca (M.S.) \\ 2 Health Canada, 414 Cooper St, Ottawa, ON K1A 0L4, Canada; joseph.byers@canada.ca \\ * Correspondence: btuana@uottawa.ca; Tel.: +1-613-562-5800 (ext. 8578)
}

\section{check for} updates

Citation: Rehmani, T.; Mlynarova, J.; Byers, J.; Salih, M.; Tuana, B.S. Specific Deletion of the FHA Domain Containing SLMAP3 Isoform in Postnatal Myocardium Has No Impact on Structure or Function. Cardiogenetics 2021, 11, 164-184. https://doi.org/10.3390/ cardiogenetics11040018

Academic Editor:

Argelia Medeiros-Domingo

Received: 31 August 2021

Accepted: 29 September 2021

Published: 4 October 2021

Publisher's Note: MDPI stays neutral with regard to jurisdictional claims in published maps and institutional affiliations.

Copyright: (c) 2021 by the authors. Licensee MDPI, Basel, Switzerland. This article is an open access article distributed under the terms and conditions of the Creative Commons Attribution (CC BY) license (https:/ / creativecommons.org/licenses/by/ $4.0 /)$.

\begin{abstract}
Sarcolemmal membrane-associated proteins (SLMAPs) belong to the superfamily of tailanchored membrane proteins known to regulate diverse biological processes, including protein trafficking and signal transduction. Mutations in SLMAP have been linked to Brugada and defective sodium channel Nav1.5 shuttling. The SLMAP gene is alternatively spliced to generate numerous isoforms, broadly defined as SLMAP1 ( 35 kDa), SLMAP2 ( 45 kDa) and SLMAP3 ( 80-95 kDa), which are highly expressed in the myocardium. The SLMAP3 isoform exhibits ubiquitous expression carrying an FHA domain and is believed to negatively regulate Hippo signaling to dictate cell growth/death and differentiation. Using the $\alpha \mathrm{MHC}-\mathrm{MerCreMer-flox} \mathrm{system} \mathrm{to} \mathrm{target} \mathrm{the} \mathrm{SLMAP}$ gene, we specifically deleted the SLMAP3 isoform in postnatal mouse hearts without any changes in the expression of SLMAP1/SLMAP2 isoforms. The in vivo analysis of mice with SLMAP3 cardiac deficiency revealed no significant changes to heart structure or function in young or aged mice without or with isoproterenol-induced stress. SLMAP3-deficient hearts revealed no obvious differences in cardiac size, function or hypertrophic response. Further, the molecular analysis indicated that SLMAP3 loss had a minor impact on sodium channel (Nav1.5) expression without affecting cardiac electrophysiology in postnatal myocardium. Surprisingly, the loss of SLMAP3 did not impact Hippo signaling in postnatal myocardium. We conclude that the FHA domain-containing SLMAP3 isoform has no impact on Hippo signaling or sodium channels in postnatal myocardium, which is able to function and respond normally to stress in its absence. Whether SLMAP1/SMAP2 isoforms can compensate for the loss of SLMAP3 in the affairs of the postnatal heart remains to be determined.
\end{abstract}

Keywords: SLMAP; $\alpha$ MHC-MerCreMer; postnatal myocardium; fork-head associated (FHA); Hippo signaling; sodium channel (Nav1.5)

\section{Introduction}

Sarcolemmal Membrane Associated Proteins (SLMAPs) belong to a superfamily of tail-anchored membrane proteins, such as junctophilin, SNAP-25 and calnexin, involved in diverse functions, including E-C coupling, neurotransmitter release and ER stress response [1]. The SLMAP gene contains alternative start sites and can alternatively splice to generate many SLMAP isoforms that are highly conserved across species and expressed in a tissue-specific and developmentally regulated manner [2]. We defined SLMAP isoforms into three main subgroups based on the molecular size of the polypeptides noted: SLMAP1 ( 35 kDa), SLMAP2 ( 45 kDa) and SLMAP3 ( 80-95 kDa) [3]. SLMAP3 is ubiquitously expressed, while SLMAP1 and SLMAP2 expression are restricted to muscle and myocardium [1,3]. All SLMAP isoforms share a common C-terminus with a single transmembrane domain, which can be alternatively spliced to target diverse subcellular membranes [4]. The SLMAPs are generally comprised of extended coiled-coils with two 
tandem leucine zippers with unique N-terminal fork head-associated domains (FHA) found in the SLMAP3 isoform [5,6]. The FHA domain is found in numerous kinases and phosphatases and acts as the regulator for phosphoprotein interactions, specifically with phospho-threonine residues [7]. Recently SLMAP3's FHA has been shown to regulate the Hippo signaling pathway via the STRIPAK (striatin interacting phosphatase and kinase) complex [8,9]. Hippo signaling is believed to regulate organogenesis, tissue homeostasis, proliferation and apoptosis, including cardiac development [10-12]. The unregulated activity of Hippo signaling in mouse myocardium can result in lethal phenotypes but can also aid in cardiac tissue repair in post-myocardial infarction or in pressure overload-induced damage [13-17]. SLMAP is believed to interact with phosphorylated MST1/2 kinases via its FHA domain and recruit STRIPAK-PP2A to dephosphorylate the transcriptional coactivators YAP/TAZ and negatively regulate Hippo to increase proliferation and repress apoptosis [18-21].

We have previously shown that endogenous cardiac SLMAPs co-localize with E-C coupling machinery, including the ryanodine receptor 2 (RyR2) and caveloin-3 in cardiomyocytes. The targeted expression of the SLMAP1 isoform in postnatal mouse myocardium leads to aberrations in electrophysiology and function due to the reduced expression of E-C coupling proteins $[22,23]$. Ishikawa and colleagues discovered a mutation in the SLMAP gene in Brugada patients, a channelopathy characterized by irregular electrical activity mainly due to abnormal expression of the sodium ion channel, $\mathrm{Na}_{\mathrm{V}} 1.5[24,25]$. The mutated SLMAP prevented the trafficking of $\mathrm{Na}_{V} 1.5$ to the cell surface in transfected HEK293 cells implicating SLMAP as a regulator of Nav1.5 protein trafficking [24]. In transgenic mice with cardiac-specific SLMAP3 expression, there was no noticeable cardiac remodeling; however, we observed a defect in cardiac function and electrophysiology due to a reduction in protein and transcript levels of Nav1.5, further implying a relationship between SLMAP and Nav1.5 [26]. Although studies have uncovered some unique properties of cardiac SLMAP, its precise molecular function needs to be fully interrogated.

Thus, our aim here was to establish a method to delete the SLMAP gene in postnatal myocardium in a conditional and temporal manner to determine its precise role in young and adult mouse hearts. In this study, we develop the procedure to establish a mouse model using the $\alpha \mathrm{MHC}-M e r C r e M e r-l o x$ method to delete the SLMAP gene in postnatal myocardium. Our strategy resulted in the specific deletion of the SLMAP3 isoform in postnatal mouse myocardium while the expression of SLMAP1 and SLMAP2 isoforms remained unaffected. We report the phenotypic assessment and any molecular impact of the specific loss of the SLMAP3 isoform in the postnatal heart under normal and $\beta$-adrenergic agonist, isoproterenol (ISO), induced stress.

\section{Materials and Methods}

\subsection{Generating Transgenic Flox-SLMAP Mouse Lines}

A targeting construct vector for SLMAP was developed using a neomycin insertion cassette. Exon 3 of the mouse SLMAP gene was flanked with loxP sites, and the vector was microinjected into embryonic stem cells that were implanted into C57BL/ 6 blastocysts. The founder (F0) flox-SLMAP mice were confirmed through genotyping and maintained through breeding with C57BL/ 6 mice (Cyagen Biosciences Inc, Santa Clara, CA, USA).

The $\alpha$ MHC-MerCreMer mice were obtained from Dr. Mona Nemer and maintained by breeding with C57BL/ 6 .

\subsection{Generating and Genotyping the SLMAP Knockout Mice}

The breeding scheme to generate a cre-lox mice line for SLMAP knockout is shown (Figure 1). Mice were genotyped by extracting genomic DNA from ear clips by boiling for $10 \mathrm{~min}$ at $95^{\circ} \mathrm{C}$ in $180 \mu \mathrm{L}$ of $50 \mathrm{mM} \mathrm{NaOH}$ per ear. The DNA solution was then neutralized using $20 \mu \mathrm{L}$ of $1 \mathrm{M}$ Tris-Cl pH 8.0. SLMAP KO/KD mice were identified by polymerase chain reaction (PCR) using DreamTaq Green PCR Master Mix 2x (Thermo Fischer Scientific, Waltham, MA, USA). Forward F2 primer (5'-CCT GGA GAG CCT CCG TGT GAG T-3') and 
reverse R2 primer (5'-GTC AAC TGC CCA ATG TAC AGA AAT AGT AAG-3') targeted loxP site 1 on the flox-SLMAP gene. Forward Cre-F (5'-ACG ACC AAG TGA CAG CAA TG-3') and reverse Cre-R primer ( $5^{\prime}$-AAC CAG CGT TTT CGT TC-3') detected the Cre gene. PCRs were visualized by Red Safe (Sigma, St. Louis, MO, USA.) staining on a $1 \%$ agarose gel.

Mice were handled in accordance with the guidelines set by Canadian Council on Animal Care, Guide to the Care and Use of Experimental Animals, 2 vols. (Ottawa, Ont.: CCAC, 1980-1993) and Animals for Research Act, R.S.O. 1990, c.A. 22. All animal protocols and procedures were approved by the Animal Care Committee of the University of Ottawa.

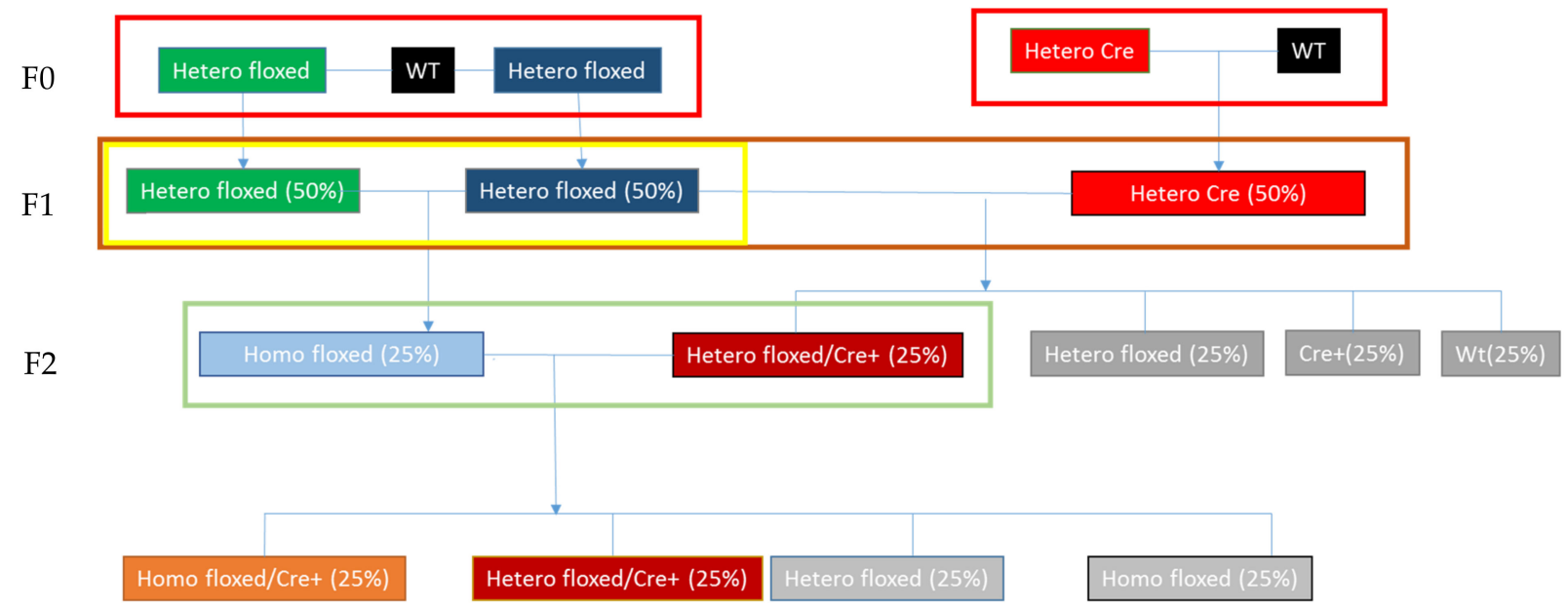

Figure 1. Breeding diagram to produce mice that contain floxed SLMAP alleles and cre. F0 generation indicates the heteroflox-SLMAP (green/dark blue) and hetero-cre (red) mice. The hetero-floxed and hetero-cre mouse lines were maintained separately with Wt. The separated hetero-flox mice offspring were bred together to generate the homo-floxed mice (blue), labeled in the F1 generation. Additionally, the F1 generation indicates the production of knock down (KD) mice (dark red; heterofloxed and hetero-cre) after breeding a hetero-cre mouse with a hetero-floxed mouse. F2 generation highlights the SLMAP3-KO (orange; homofloxed and cre) mice generated by breeding a homo-floxed animal with a KD animal.

\subsection{Activation of Cre and Assessing Recombination of Floxed SLMAP}

Tamoxifen (Sigma-Aldrich, St. Louis, MO, USA) was administered to animals to activate MerCreMer. A total of $500 \mu \mathrm{g}$ of tamoxifen was dissolved into $10 \mathrm{~mL}$ of peanut oil (Sigma-Aldrich, St. Louis, MO, USA.), aliquoted into 10 tubes and frozen at $-20{ }^{\circ} \mathrm{C}$ for long-term storage. Tamoxifen was injected intraperitoneally in 5-week-old animals at a dose of $30 \mu \mathrm{g}$ tamoxifen/g of animal for 3 days. Tamoxifen treated hearts were analyzed by PCR genotyping as described above. The forward primer F2 (5'-CCT GGA GAG CCT CCG TGT GAG T-3') and reverse primer R1 (5'-GGA GAG ACT ATC ACA GCC ACA GGA-3'), coding for sequences between loxP sites, exon 3 and intron sequences, were used for amplification.

\subsection{Protein Isolation from Mouse Heart}

The hearts of adult mice (8-12 weeks of age) were collected after CO2 euthanasia and immediately frozen at $-80^{\circ} \mathrm{C}$. Each heart was washed with ice-cold $1 \times$ phosphatebuffered saline (PBS) and homogenized using a Fisher handheld Maximizer homogenizer (Thermo Fisher Scientific, Waltham, MA, USA.) in ice-cold lysis buffer ( $1 \mathrm{mM}$ ethylene glycol tetraacetic acid (EGTA), $1 \mathrm{mM}$ ethylenediaminetetraacetic (EDTA), $20 \mathrm{mM}$ Tris base, $1 \%$ Triton, $150 \mathrm{mM}$ sodium chloride, $1 \times$ complete mini EDTA-free protease inhibitor cocktail (Roche, Basel, Switzerland) and $1 \times$ PhosSTOP (Roche, Basel, Switzerland). The suspension was centrifuged for $15 \mathrm{~min}$ at $12,000 \times g$ to separate the proteins from the cell debris. The supernatant containing heart protein lysate was collected in Eppendorf tubes and stored in a freezer at $-80^{\circ} \mathrm{C}$. 


\subsection{SDS-PAGE and Western Blotting}

A total of 10-20 $\mu \mathrm{g}$ of protein lysate was loaded in each well of a 5-15\% SDS-PAGE gel. The gels were transferred overnight on a polyvinylidene fluoride (PVDF) membrane (Bio-Rad, Hercules, CA, USA) in a buffer containing $25 \mathrm{mM}$ Tris, $190 \mathrm{mM}$ Glycine and $20 \%$ methanol. All membranes were blocked at room temperature for $1 \mathrm{~h}$ in Tris-buffered saline (TBST) containing $1 \mathrm{M}$ Tris, $290 \mathrm{mM} \mathrm{NaCl}, 0.1 \%$ Tween 20, pH 7.4 and $5 \%$ nonfat dry milk. The primary antibodies (listed in Table 1) were incubated overnight at $4{ }^{\circ} \mathrm{C}$ with $5 \%$ bovine serum albumin (BSA). The membranes were washed 5 times for $5 \mathrm{~min}$ each in TBST prior to adding the appropriate horseradish peroxidase-labeled secondary antibody (Jackson Immuno Research, West Grove, PA, USA) at a 1:10,000 dilution in TBST with $5 \%$ nonfat dry milk. The membranes were shaken slowly at room temperature for 1 $\mathrm{h}$ while incubating with secondary antibody, followed by 5 washes for 5 min each with TBST. The membranes were treated with a BioRad western blotting kit (Bio-Rad, Hercules, USA) and developed using ChemiDoc machines (Bio-Rad, Hercules, USA). The bands were quantified by densitometry using Image Lab software v.6.0.0 (Bio-Rad, Hercules, CA, USA). The membranes were stripped ( $25 \mathrm{mM}$ glycine, $10 \% \mathrm{SDS}$ and $\mathrm{pH} 2.2 \mathrm{in} \mathrm{dH} 2 \mathrm{O}$ ) and reprobed with different antibodies. When using stain-free technology, stain-free gels (Bio-Rad, Hercules, CA, USA) and low-fluorescence PVDF membranes (Bio-Rad, Hercules, CA, USA) were used.

Table 1. List of antibodies used in this study. All antibodies used in this study are listed with the corresponding distributor, catalog number and dilution used for western blot.

\begin{tabular}{ccc}
\hline Antibody & Manufacturer & Dilution \\
\hline SLMAP & Novus Biologicals (NBP1-81397) & $1: 1000$ \\
Nav1.5 & Alomone Labs (ASC-005) & $1: 600$ \\
Striatin & BD Transduction Laboratories (610838) & $1: 1000$ \\
PP2A-C $\alpha / \beta$ subunit & Santa Cruz Biotechnology (sc-80665) & $1: 200$ \\
PP2A-A $\alpha$ subunit & Millipore Sigma (07-250) & $1: 600$ \\
Phospho-YAP (S127) & Cell Signaling Technology (13008S) & $1: 1000$ \\
YAP & Cell Signaling Technology (14074S) & $1: 1000$ \\
Phospho-MST1/2 (T183/180) & Cell Signaling Technology (49332S) & $1: 1000$ \\
KRS2(MST1) & Santa Cruz (sc-515051) & $1: 200$ \\
\hline
\end{tabular}

\subsection{Quantitative PCR}

The total mRNA from mouse hearts was extracted using the RNeasy Fibrous Tissue Kit (Qiagen, Hilden, Germany). The concentration and purity of the obtained mRNA were determined by measurement of $260 / 230 \mathrm{~nm}$ absorbance ratio and 280/260 absorbance ratio [27] using NanoDrop 2000 UV-Vis Spectrophotometer (Thermo Fisher Scientific, Waltham, MA, USA). RNA was used as a template to generate cDNA using SuperScript II reverse transcription protocol following the manufacturer's guidelines (Thermo Fisher Scientific, Waltham, MA, USA). RT-qPCR was carried out using a BioRad CFX96 system and Luna Universal qPCR mater mix according to the manufacturer's instructions. Equal amounts of cDNA were utilized in real-time PCR using primers for SLMAP3 CDNA (SLPNF: 5'-GGA ATT CGA TGC CGT CAG CCT TGG C-3', 558-R: 5' -TTG CTC GTC TTG TGA TCA AAC CAG-3'), total SLMAP cDNA (GTY-F: 5'-GAA AAG CCT ATC GAA ATC AAG TTG-3', GTY-R: 5'-ACC TTC TTA AGC TCT TCT TGC AAA G-3') and 18S Ribosome control (forward: 5'-AAT ACA TGC CGA CGG GCG CT-3', Reverse: 5' AGT GGG TAA TTT GCG CGC CT- $3^{\prime}$ ). The fold change was calculated using the $\Delta \Delta \mathrm{Ct}$ method.

\subsection{Echocardiography}

All echocardiographic analysis was performed using the VEVO 2100 system (FUJIFILM VisualSonics, Toronto, Canada). Adult mice were anesthetized using $2 \%$ isoflurane and strapped onto a heated pad facing upwards, exposing the thoracic cavity. A $40 \mathrm{MHz}$ probe was used to capture short-axis B-mode and M-mode images of the left ventricle. 
VEVO v1.6 software (FUJIFILM VisualSonics Toronto, Canada) was utilized for measuring $\mathrm{LV}$ wall thickness and inner diameters in diastole and systole. The formulas used by the VEVO v1.6 software to calculate EF, FS, LV mass and LV Vol;d/s are listed below:

$$
\begin{aligned}
& \mathrm{EF}: 100 \times\left(\frac{\mathrm{LV} \mathrm{Vol} ; \mathrm{d}-\mathrm{LV} \mathrm{Vol} ; \mathrm{s}}{\mathrm{LV}} \mathrm{Vol} ; \mathrm{d}\right) \\
& \text { FS : } 100 \times\left(\frac{\text { LVID } ; \mathrm{d}-\text { LVID;s }}{\text { LVID }} ;\right) \\
& \text { LV mass : } 0.8 \times 1.053 \times\left((\text { LVID; } \mathrm{d}+\text { LVPW; } \mathrm{d}+\text { IVS; } \mathrm{d})^{3}-\mathrm{LVID} ; \mathrm{d}^{3}\right) \\
& \text { LV vol; d/s : }((7.0 /(2.4+\text { LVID; } d ; s)) \times \operatorname{LVID} ;(d ; s) 3
\end{aligned}
$$

\subsection{Isoproterenol Delivery}

Isoproterenol (Sigma-Aldrich, St. Louis, MO, USA) delivery was mediated through the subcutaneous implantation of miniosmotic pumps (2001D Alzet, Cupertino, CA, USA) in 8week-old mice. $0.9 \%$ saline or isoproterenol (dissolved in $0.9 \%$ saline) was delivered at a rate of $30 \mu \mathrm{g} / \mathrm{g} / \mathrm{d}$ per animal. Cardiac function was analyzed through echocardiography before pumps were implanted and after seven days. After a week of isoproterenol administration, the animals were euthanized, and the hearts were collected for histological analysis.

\subsection{Histological Analysis}

Hearts that were iso-treated were extracted from animals and fixed using 10\% Neutral Buffered Formalin (Thermo Fisher Scientific, Waltham, MA, USA.). After fixing for $48 \mathrm{~h}$, the hearts were sectioned $4 \mu \mathrm{m}$ longitudinally per section. Sectioned hearts were stained with Masson's trichrome to visualize the myocardium, nuclei and collagen.

\subsection{Electrocardiography}

The mice were anesthetized with 2.5\% isoflurane. A 6-lead surface ECG was recorded after a 5-min stabilization period of mice in the anesthetized state. ECG intervals and heart rate were analyzed manually from 6-18 most stable waves selected from a 2-min recorded stream. Analysis was blind to the genotype. Intervals were defined as follows: PP duration from the beginning of the $\mathrm{P}$ wave to the end, where the $\mathrm{P}$ wave returns to the isoelectric line, PR segment from the end of the $P$ wave to the beginning of $R$ wave, $P R$ interval from the beginning of $\mathrm{P}$ wave to the beginning of the $\mathrm{R}$ wave, $\mathrm{QRS}$ duration from the beginning of the $\mathrm{R}$ wave to the point where the negative $S$ wave returns to the isoelectric line and QT interval from the beginning of the $\mathrm{R}$ wave to the point where the negative or positive $\mathrm{T}$ wave returns to the isoelectric line. The $Q$ wave was not visible. QT interval correction was based on Mitchell [28] using the following formula: QTc $=\mathrm{QTo} /(\mathrm{RRo} / 100) 1 / 2$. P, $\mathrm{R}$ and $\mathrm{S}$ wave amplitudes were quantified as the distance between the peak of the wave and the isoelectric line connecting the end of the $\mathrm{P}$ wave and the beginning of the $\mathrm{R}$ wave. Electrocardiograms were recorded using IOX2.4.2.6 (EMKA Technologies, Paris, France). RR interval, heart rate and wave amplitudes ( $\mathrm{P}, \mathrm{R}$, and $\mathrm{S}$ ) were analyzed by ecgAUTO v2.5.1.18 software (EMKA Technologies, Paris, France).

\subsection{Statistical Analyses}

The statistical analysis was performed using GraphPad Prism software version 8 for Windows (GraphPad Software, La Jolla, CA, USA.). All comparisons between wildtype and knockdown or knockout groups were analyzed using a two-tailed Student's $t$-test. To analyze all three groups, we utilized ANOVA one-way analysis to evaluate the significance. All values and points on graphs represent mean values obtained from multiple experiments. All error bars presented in graphs are represented using the standard error of the mean. All sample size values $(n)$ represent biological replicates. 


\section{Results}

\subsection{Producing the $\alpha M H C-M e r C r e M e r-F l o x-S L M A P$ Mice}

The mouse SLMAP gene is approximately 122 kilo-base pairs (bp) in length and contains 24 exons. Exons 2, 3, 10 and 16 are believed to contain putative start sites, and seven exons $(11,12,13,14,17,23$ and 24$)$ can alternatively splice to generate additional SLMAP isoforms (Figure 2) based on our lab findings and proteomic/genomic profiling databases [29-31]. SLMAP3 variants are generated by alternate start sites found at exon 2 or 3 , and the alternative splicing of exons 11, 12, 13, 14 and 17 (Figure 2). SLMAP2 and SLMAP1 isoforms are generated by putative start sites at exon 10 and exon 16, respectively (Figure 2). SLMAP2 variants are similarly generated by alternative splicing of exons 11, 12, 13, 14 and 17, while SLMAP1 variants have an alternative exon 17 (Figure 2). All SLMAP isoforms house an alternative transmembrane domain that is generated by mutually exclusive splicing of exon 23 or 24 (Figure 2). We predicted that promoter sequences for SLMAP would reside upstream of exon 2; therefore, we targeted the excision of exon 3 to prevent the translation of functional SLMAP as it would cause a frameshift mutation. To generate the cardiac-specific postnatal knockout of SLMAP, the cre-lox system was chosen as we could manipulate the temporal and spatial targeting by using the $\alpha \mathrm{MHC}-\mathrm{MerCreMer}$ mice, ensuring that cre recombinase is expressed specifically in the myocardium and will remain dormant unless activated by an estrogen analog, tamoxifen [32,33]. To target exon 3 , in the mouse SLMAP gene, loxP sites were introduced to flank exon 3 . The floxedSLMAP DNA was microinjected into embryonic stem cells that were transplanted into fertilized mouse ovum (detailed in materials and methods) [34]. Floxed-SLMAP mice were crossbred with $\alpha$ MHC-MerCreMer mice to generate offspring that would contain both a floxed-SLMAP and cre transgene to undergo a knockout (KO) or knockdown (KD) of SLMAP in postnatal mouse hearts (detailed in materials and methods) [35].

PCR genotyping of ear clippings from individual mice determined the presence of floxedSLMAP and/or $\alpha$ MHC-MerCreMer. Primers F2/R2 were used to identify floxed-SLMAP by detecting loxP site 1 and primers $\mathrm{CreF} / \mathrm{CreR}$, detected $\alpha M H C-M e r C r e M e r$ as modeled in Figure 3A. The flox-SLMAP F2/R2 PCR product was $424 \mathrm{bp}$ in length, whereas wildtype SLMAP was $282 \mathrm{bp}$ in length (Figure 3B). Genotyping with CreF/CreR generated a $425 \mathrm{bp}$ amplicon denoting a $\alpha \mathrm{MHC}-\mathrm{MerCreMer}{ }^{+}$mouse (Figure $3 \mathrm{~B}$ ). PCR products ran on separate lanes on $1 \%$ agarose gel to separate and size DNA amplicons to determine the presence of transgenes in each animal (Figure 3B). The lane labeled KO presented two alleles of flox-SLMAP (one DNA band at $424 \mathrm{bp}$ ) and the $\alpha \mathrm{MHC}-\mathrm{MerCreMer}$ transgene, signifying a KO mouse (Figure 3B). The KD lane presented one flox-SLMAP allele (424 bp), one wt-SLMAP allele (284 $\mathrm{b}$ bp) and the $\alpha \mathrm{MHC}-\mathrm{MerCreMer}$ transgene, signifying a KD mouse (Figure $3 \mathrm{~B}$ ). Finally, the lane that had only one transgene or none signifies a wildtype (Wt) animal, as both a flox and a cre are essential for DNA modification to take place (Figure 3B) [36]. 

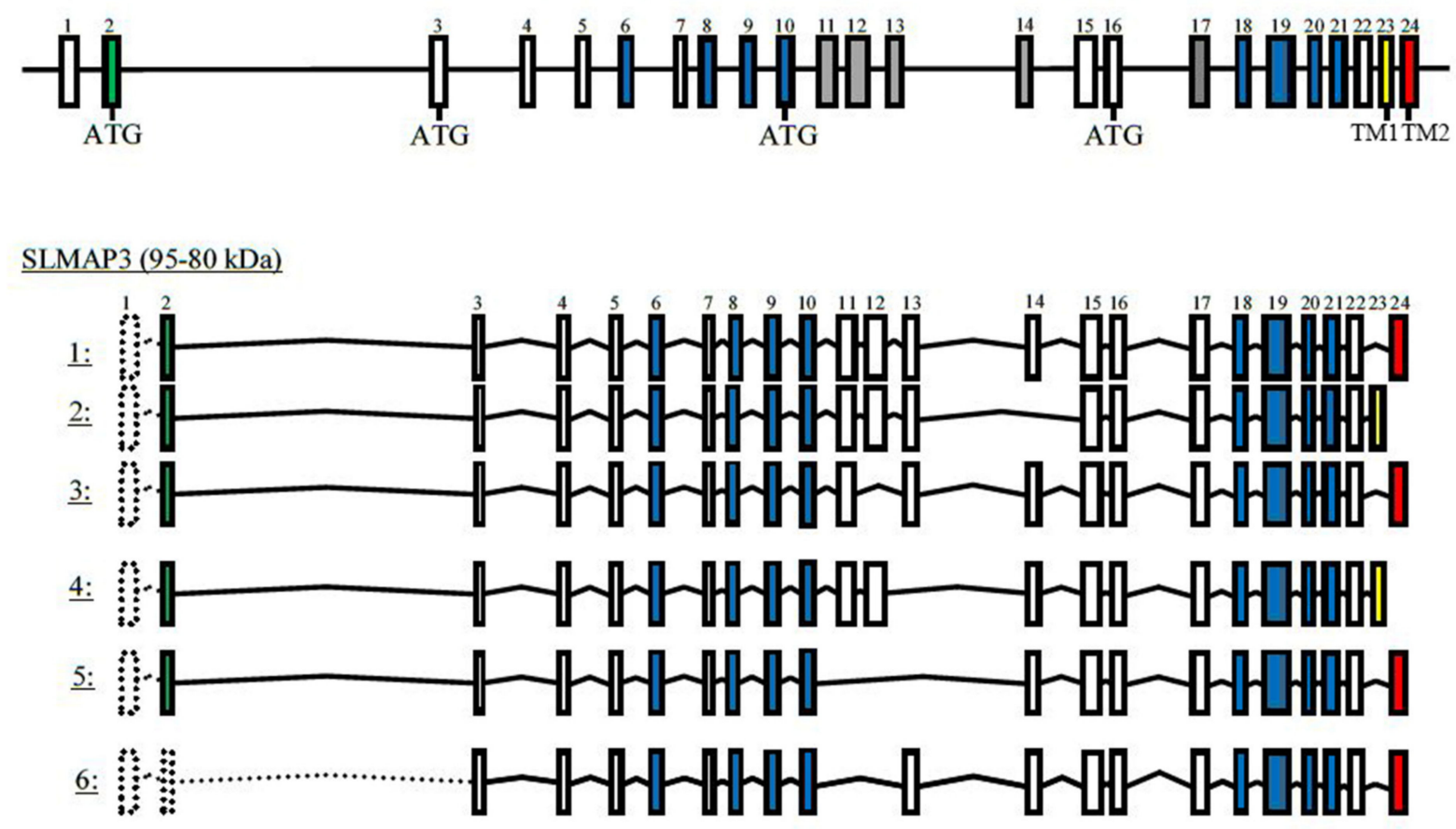

\section{$\underline{\operatorname{SLMAP} 2(45 \mathrm{kDa})}$}

7:

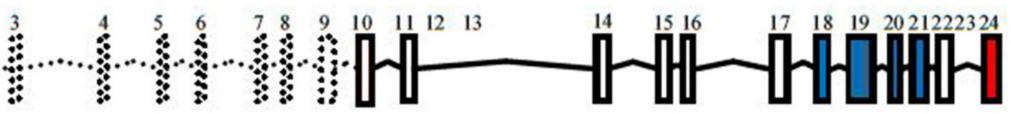

8:

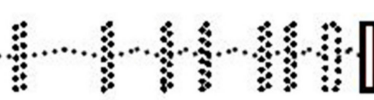

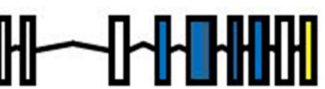

SLMAP1 (35kDa)

9:

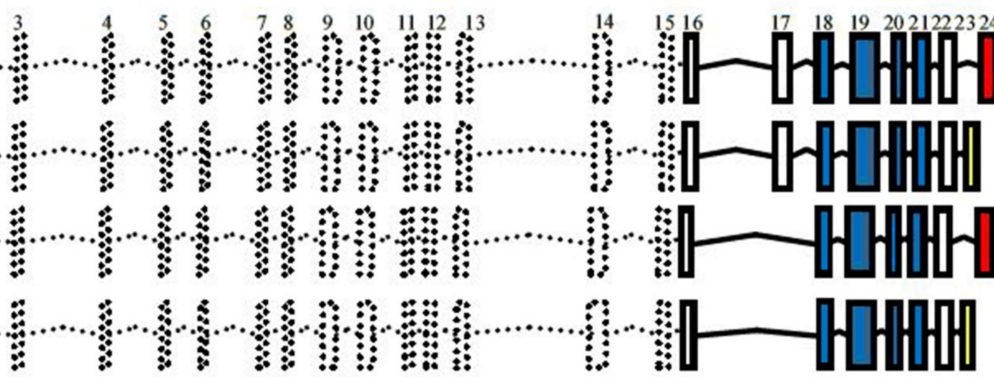

Figure 2. Intron-exon junction of mouse SLMAP gene and its splice variants. SLMAP consists of 24 exons (black) and alternatively splices to generate numerous isoforms grouped as three isoforms, SLMAP1 (35 kDa), SLMAP2 (45 kDa) and SLMAP3 (80-95 kDa). The start codon (ATG) for SLMAP3, SLMAP2 and SLMAP1 are found at exon 2/3, 10 or 16, respectively. Alternative splicing of exons 11, 12,13, 14 and 17 results in each isoform presenting unique variants. Exon 2 (green) codes for the FHA domain. Exons 6, 8, 9, 10, 18, 19, 20 and 21 (blue) code for the coiled-coil LZ domains. Mutually exclusive exons 23 (yellow) and 24 (red) code for transmembrane domain 1 (TM1) or transmembrane domain 2 (TM2). 
A.

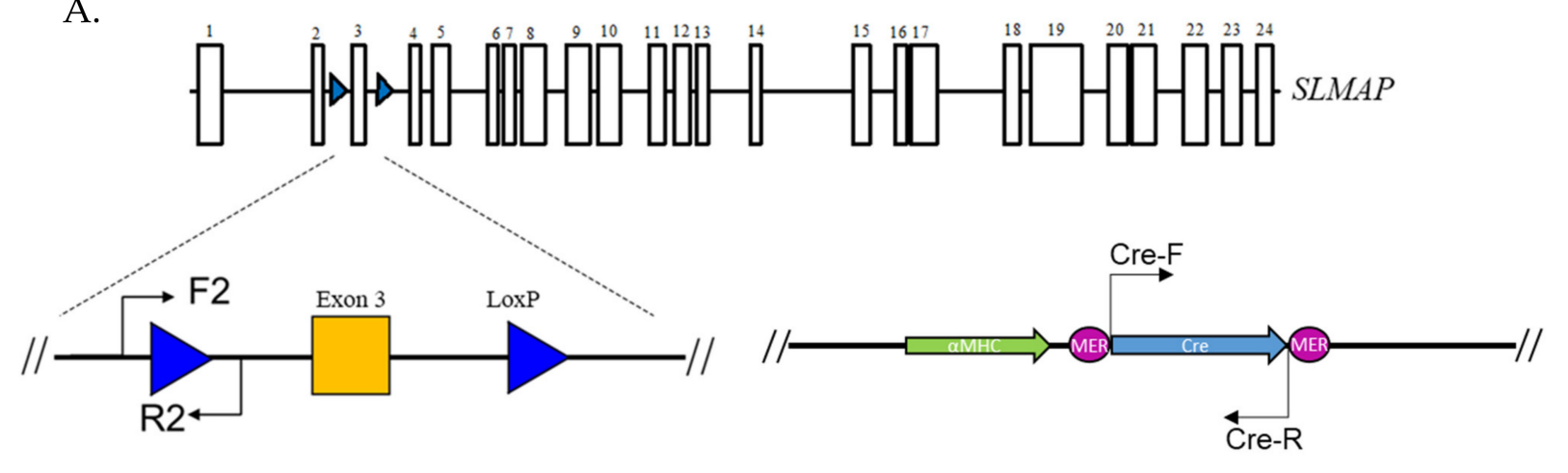

B.

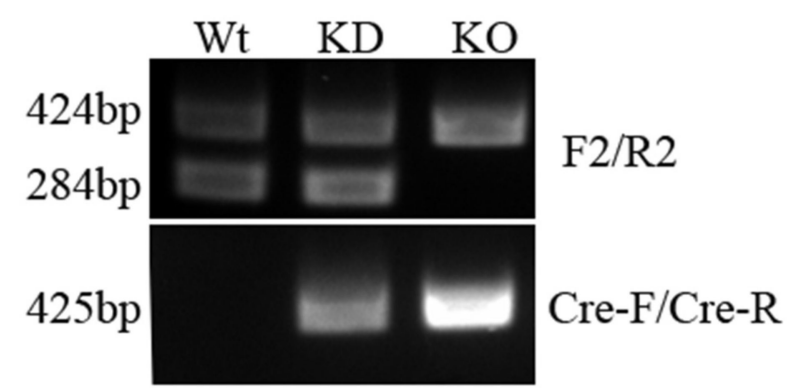

Figure 3. PCR genotyping detects floxed-SLMAP and $\alpha \mathrm{MHC}-\mathrm{MerCreMer}$ gene in mice. (A) Schematic representation of primers F2/R2 identifying loxP site 1 on flox-SLMAP gene and primers Cre-F/Cre-R identifying $\alpha$ MHC-MerCreMer gene. (B) PCR amplicons run on separate lanes on a 1\% agarose gel labeled Wt, KD or KO identifying a wildtype, knocked-down or knockout mouse heart, respectively.

\subsection{Cleavage of Floxed-SLMAP by Activating $\alpha M H C-M e r C r e M e r$}

After generating flox-SLMAP / $\alpha$ MHC-MerCreMer ${ }^{+}$mice, we wanted to ensure loxP sites flanking SLMAPs exon 3, and surrounding introns were correctly targeted. This was assessed through PCR amplification of DNA isolated from tamoxifen-treated hearts of Wt and KD mice. Studies have shown that the administration of $30 \mu \mathrm{g}$ of tamoxifen/g of body weight for 3 days via intraperitoneal injections is the most effective method for inducing MerCreMer activity because it results in the highest recombination efficiency [33,37]. After tamoxifen treatment, we extracted DNA from KD and Wt hearts and performed PCR using primers F2/R1, targeting the sequence between the two loxP sites, modeled in Figure 3A. Excision of the floxed region would reduce the F2/R1 product to $400 \mathrm{bp}$ while the unaffected SLMAP gene's F2/R1 product would remain $1400 \mathrm{bp}$ in size (Figure $4 \mathrm{~A}$ ). PCR products ran individually on $1 \%$ agarose gels to separate and size the DNA amplicons from Wt and KD hearts (Figure 4B). Amplicons from KD hearts produced bands at 1400 and $400 \mathrm{bp}$, demonstrating that only floxed SLMAP allele is targeted by the active cre enzyme (Figure $4 \mathrm{~B}$ ). Floxed-SLMAP / $\alpha$ MHC-MerCreMer- $(\mathrm{Wt}$ ) hearts produced an amplicon at $1400 \mathrm{bp}$, signifying that cre is necessary to cleave floxed genes (Figure 4B). Therefore, the activation of $\alpha \mathrm{MHC}-\mathrm{MerCreMer}$ via tamoxifen successfully excised loxP flanked exon 3 from the floxed-SLMAP gene. 
A.

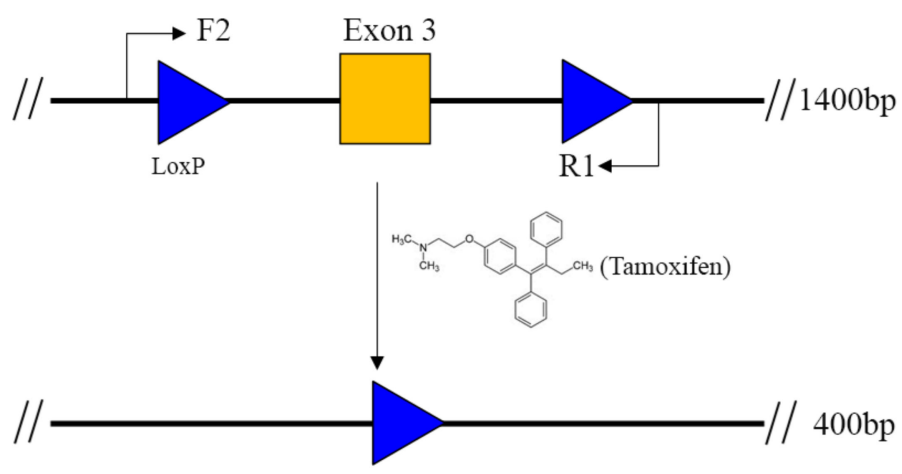

B.

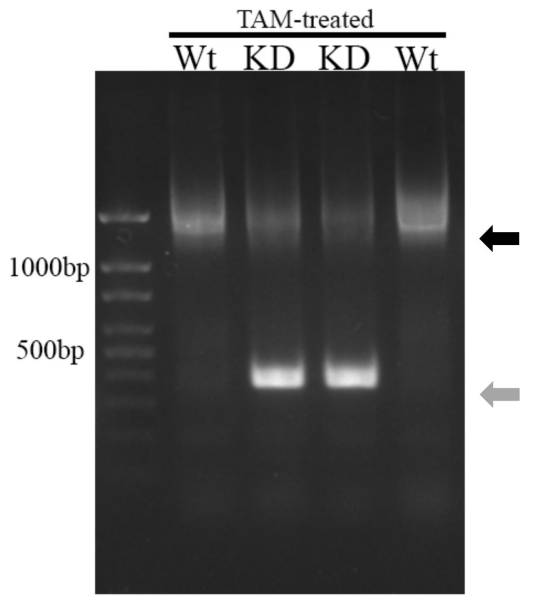

Figure 4. Administration of tamoxifen cleaves exon 3 of floxed SLMAP gene by activating $\alpha$ MHC-MerCreMer in mouse hearts. (A) Schematic representing cleavage of exon 3 in a KD mouse after treatment with tamoxifen. (B) PCR genotyping of hearts treated with tamoxifen (TAM) using primers F2/R1. Wt lanes indicate unaltered SLMAP, which results in a $1400 \mathrm{bp}$ band. KD lanes result in two bands being present, a cleaved SLMAP band (400 bp; grey arrow) and an unaltered SLMAP gene (1400 bp; black arrow).

\subsection{Protein and Transcript Expression of SLMAPs in Wt, KD and KO Mice}

Next, we wanted to assess the impact on SLMAP protein levels in KD and KO mice. Adult mice were tamoxifen-treated and left for a minimum of three weeks before cardiac lysates were evaluated for protein expression. Western blot analysis on mice designated KD had a significant reduction in the expression of SLMAP3 $(-45.81 \% \pm 9.58 \%, \rho<0.05$, $n=6$ ) relative to the $\mathrm{Wt}$ (Figure $5 \mathrm{~A}$ ); however, no changes were noted in the expression of SLMAP1 or SLMAP2 isoforms. In KO mice, western blot analysis indicated the total loss of SLMAP3 protein $(-89.31 \% \pm-14.50 \%, \rho<0.001, n=10)$, but there were no changes noted in the expression of SLMAP1 $(1.91 \% \pm 31.94 \%, \rho=0.955, n=10)$ or SLMAP2 $(12.86 \% \pm$ $35.02 \%, \rho=0.74, n=10$ ) in KO hearts when compared to Wt littermates (Figure 5A). Further quantification of western blot data indicated that the expression of SLMAP2 (11.07x greater, $\rho<0.05, n=3)$ and SLMAP1 (22.91x greater, $\rho<0.05, n=3)$ was much higher compared to SLMAP3 expression in Wt adult mouse myocardium (Figure 5B).

The transcript levels of the SLMAP isoforms in tamoxifen-treated postnatal mouse hearts amplified using PCR with specific primers, SLPN-F/558-R and GTY-F/GTY-R, target exons at the $5^{\prime}$ end, sequences that are present in SLMAP3 but absent in SLMAP1 and SLMAP2 (Figure 2) [1]. We observed a significant decrease in SLMAP3 transcripts in the $\mathrm{KD}(-55.22 \% \pm 27 \%, n=19, \rho<0.001)$ and $\mathrm{KO}(-93.47 \% \pm 31.20 \%, n=13, \rho<0.001)$ hearts (Figure 5B). SLMAP1 and SLMAP2 sequences are conserved in SLMAP3; therefore, we are unable to amplify these distinct isoforms individually. Instead, we used GTY-F/GTY-R, a primer that targets all three isoforms, to determine if there are any differences in total SLMAP mRNA expression. Total SLMAP expression in KD $(-1.29 \% \pm 38.35 \%, n=10$, $\rho=0.759)$ and $\mathrm{KO}(-34.12 \% \pm 40.71 \%, n=9, \rho=0.759)$ hearts revealed no significant differences compared to Wt (Figure 5B). Therefore, targeting exon 3 of the SLMAP gene nullifies SLMAP3 expression but not the abundantly expressed SLMAP1 or SLMAP 2 mRNA [1]. 

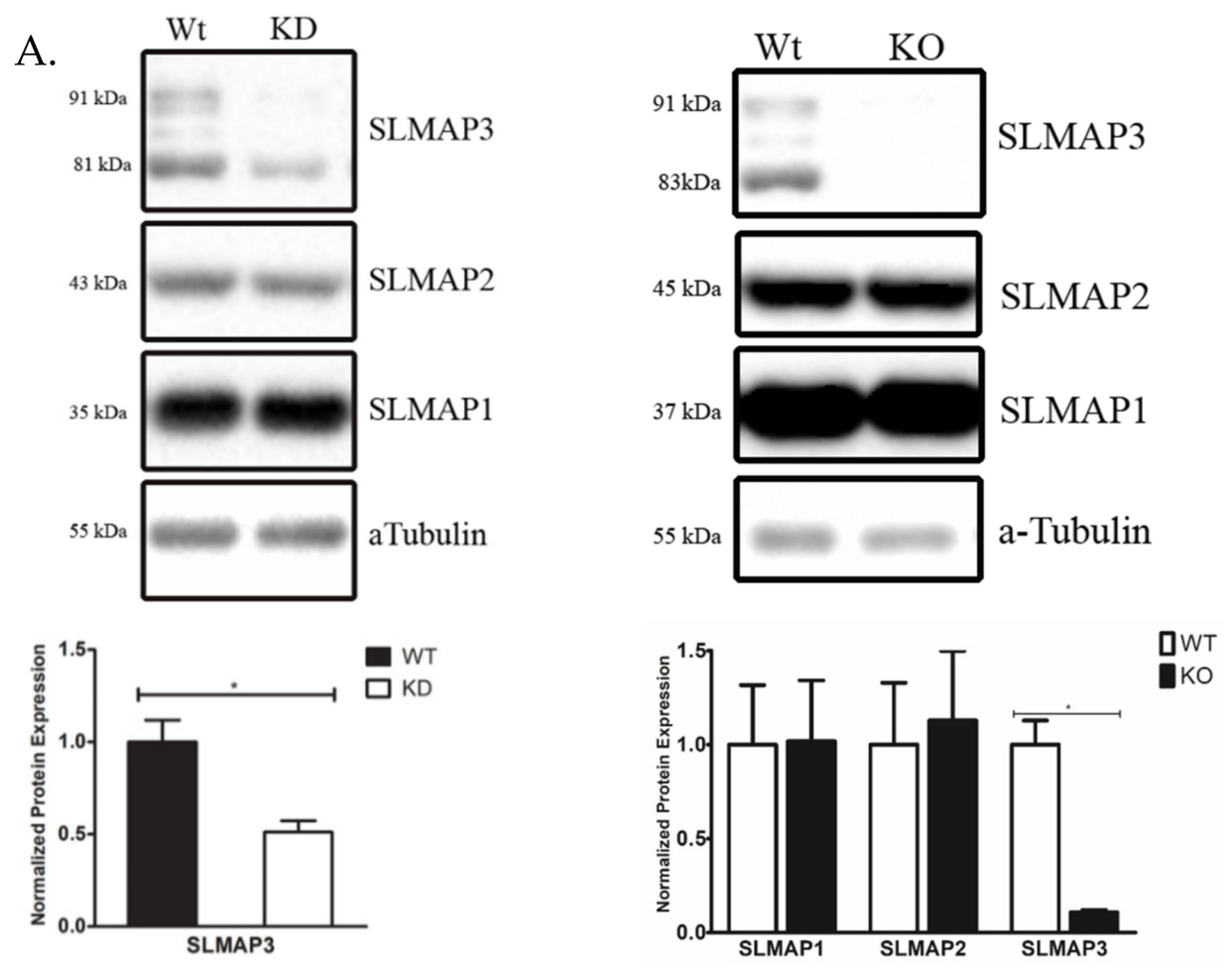

B.

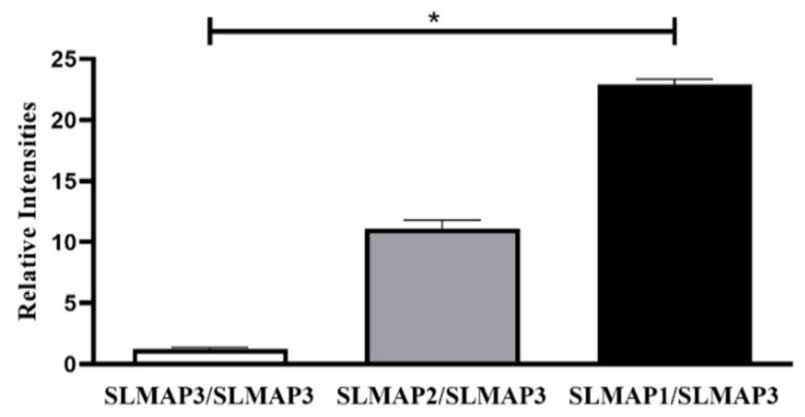

C.
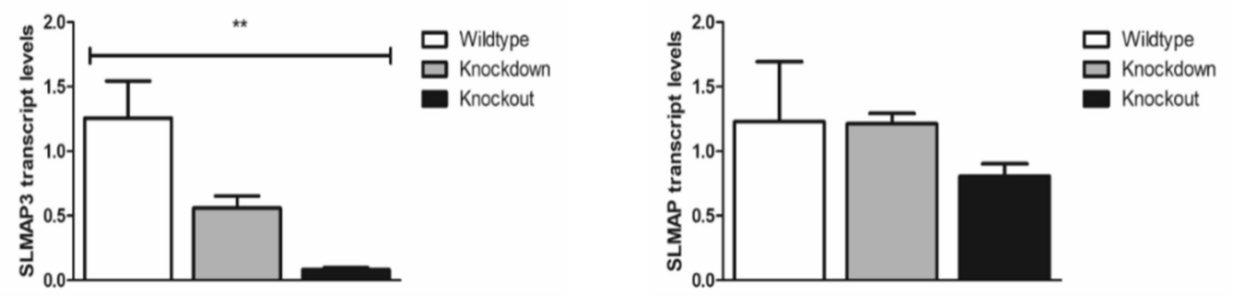

Figure 5. Protein and transcript expression of SLMAP isoforms in targeted mice. (A) Western blot with anti-SLMAP for protein expression in $\mathrm{Wt}, \mathrm{KD}$ and $\mathrm{KO}$ hearts. Bar graphs represent normalized protein expression evaluated via densitometry analysis by using $\alpha$-Tubulin as a loading control. $n=10 .{ }^{*} \rho<0.05, \rho$ value was calculated using a non-paired two-way $t$-test. (B) Relative intensity of protein band ratios of SLMAP2 and SLMAP1 normalized to SLMAP3 from western blots in Wt hearts. $n=3 * \rho<0.05$. (C) Transcript levels for SLMAP3 and total SLMAP in KD and KO hearts compared to Wt. Fold change was calculated using 18S-Ribosome as control. $n=9$. ${ }^{* *} \rho<0.001, \rho$ value was calculated using ANOVA statistical analysis. 


\subsection{Cardiac Structure and Function of SLMAP3-Deficient Hearts}

Although only SLMAP3 expression was nullified in our cre-lox model, we asked about its impact in adult mouse hearts since SLMAP3 has been implicated in many roles [22-24,26]. Transthoracic echocardiography (ECHO) was performed on 5-week post-tamoxifen (post-TAM) mice biweekly for 19 weeks to observe any changes to cardiac structure and function by measuring left ventricle (LV) wall thickness (intraventricular septum (IVS) and left ventricular posterior wall (LVPW)) and the left ventricular intradiameter (LVID) during systole and diastole. These measurements were used to calculate cardiac function, including the ejection fraction (EF) and fractional shortening (FS), which measure a percentage of cardiac output and LV muscle contraction, respectively [38]. ECHO did not reveal any structural abnormalities in the young (5-week post-TAM) or old (24-week post-TAM) SLMAP3-deficient hearts when compared to $\mathrm{Wt}$ (Figure 6A). Further, analysis of LV walls revealed no significant changes to IVS or LVPW wall thickness during systole or diastole in young or old SLMAP3 deficient hearts when compared to wildtype (Supplementary Table S1). Further cardiac functional analysis indicated no significant differences in EF or FS in SLMAP3-deficient hearts (Figure 6B). Left ventricle mass to body weight comparison indicated no significant differences between KD, KO mice $(n=6, \rho=0.5826)$ and wildtype (Figure $6 \mathrm{C}$ ). The only difference noted between the age groups was an increase in LV mass that was likely due to age-related cardiac growth, which was similar among $\mathrm{Wt}$ and SLMAP3 null hearts (Supplementary Table S1). Therefore, a deficiency of SLMAP3 did not impact cardiac structure or function in the postnatal young or adult mouse hearts. 
A.
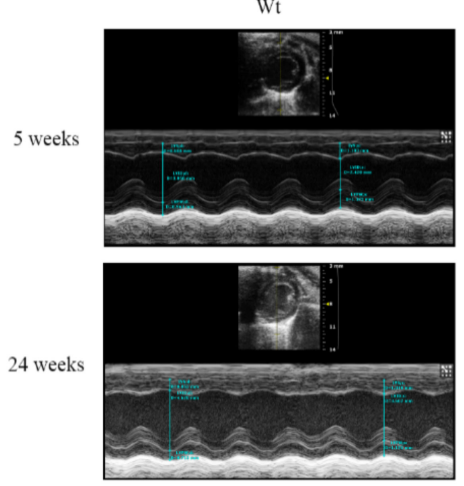

$\mathrm{KD}$
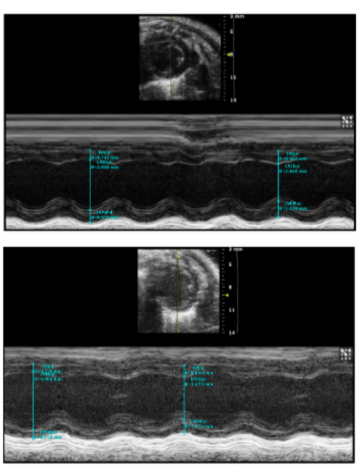

KO

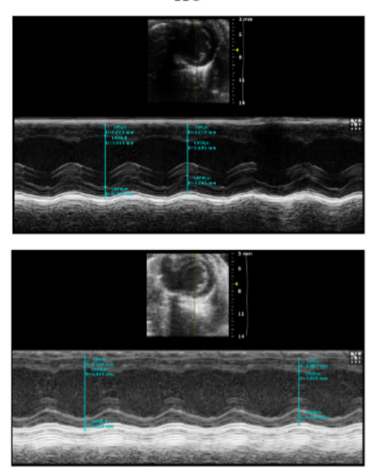

B.
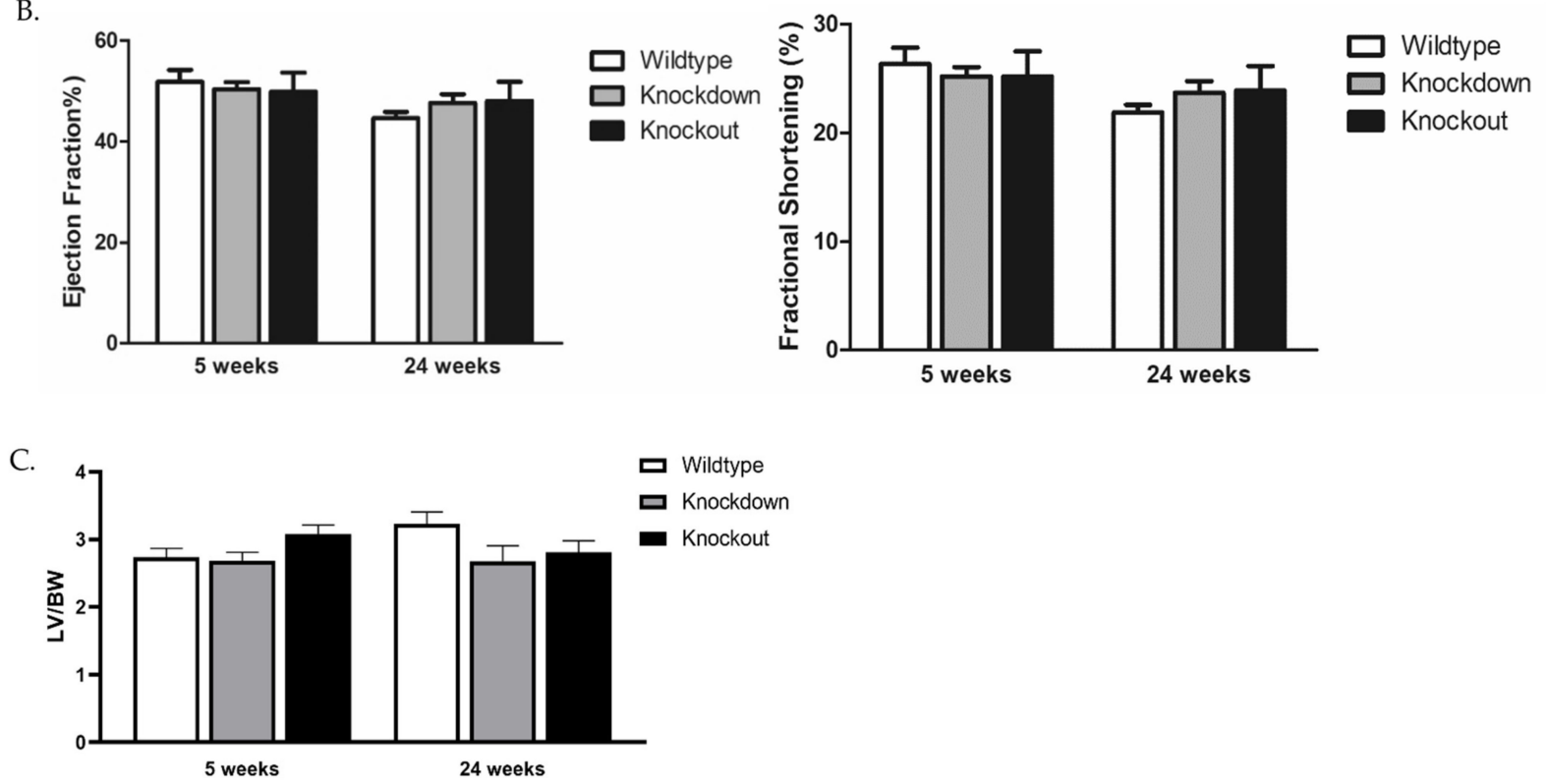

Figure 6. Echocardiography and left ventricular function of SLMAP3-deficient hearts. (A) Representative short-axis m-mode images of $\mathrm{Wt}, \mathrm{KD}$ and $\mathrm{KO}$ hearts at 5 weeks and 24 weeks post tamoxifen. (B) Bar graphs represent cardiac functional analysis via ejection fraction (\%) and fractional shortening (\%) of young and aged SLMAP3-deficient hearts. (C) The bar graph represents left ventricle mass to body weight ratio ( $\mathrm{LV} / \mathrm{BW})$ in $\mathrm{Wt}, \mathrm{KD}$ and $\mathrm{KO}$ mice. $n=6$.

\subsection{Impact of Isoproterenol Induced Stress on SLMAP3-KO Hearts}

Our next aim was to administer cardiac stressor isoproterenol (ISO) to cardiac-depleted SLMAP3 mice to evaluate the impact of stress on the myocardium [39]. ISO is a non-selective $\beta$-adrenergic agonist that can induce pathological cardiac hypertrophy [40]. Seven-day delivery of ISO (30 ug/g) or saline-control was mediated through mini-osmotic pumps that were subcutaneously implanted in tamoxifen-treated eight-week-old adult mice that were genotyped $\mathrm{KO}$ or Wt. ECHOs were performed before pumps were implanted (preISO) and seven days after implantation (postISO). PostISO ECHOs revealed a significant change in LV wall thickness in $\mathrm{KO}$ and Wt hearts (Figure 7A). LV walls, IVS ( 25\% increase, $n=7, p<0.05)$ and LVPW $(\sim 20 \%$ increase, $n=7, \rho<0.05)$ were significantly thicker in postISO mice in both Wt and KO hearts when compared to their preISO measurements (Figure 7B). Unsurprisingly, the weight of the hearts increased $(25 \%$ increase, $n=7, \rho<0.05)$ as a consequence of cardiac hypertrophy. The similarity in heart weight and dimensions between the two iso-treated groups indicates the hypertrophic stress response is identical between the SLMAP3-KO and wildtype hearts (Supplementary Table S2).

Hearts dosed with saline or ISO underwent histological analysis to observe changes to all chambers of the myocardium. Wt and $\mathrm{KO}$ hearts were fixed, sectioned longitudinally 
and stained with Masson's trichrome. Masson's trichrome stains the cardiomyocytes, nucleus and collagen; thus, we are able to visualize the myocardium and detect fibrotic cells. After a seven-day ISO treatment, SLMAP3-KO hearts displayed no obvious structural differences, nor did we detect any evidence of fibrosis when compared to Wt (Figure 8).
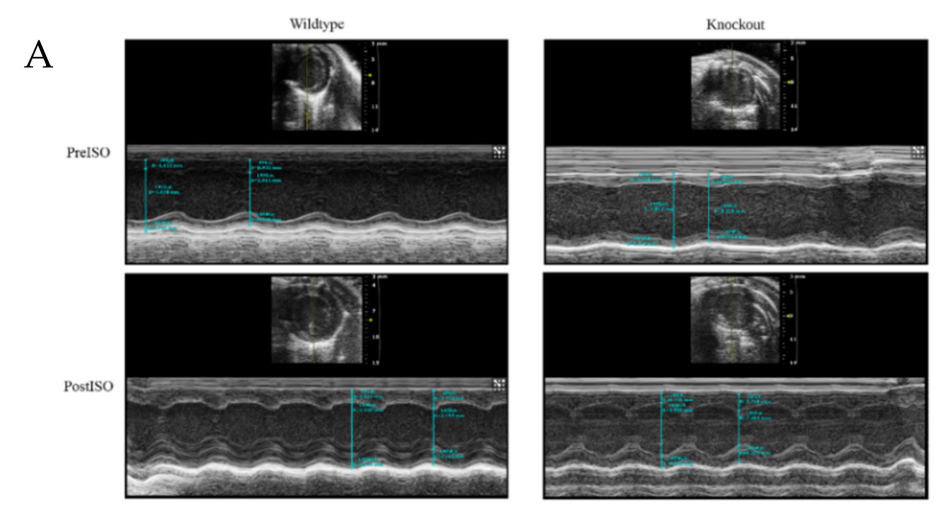

B.
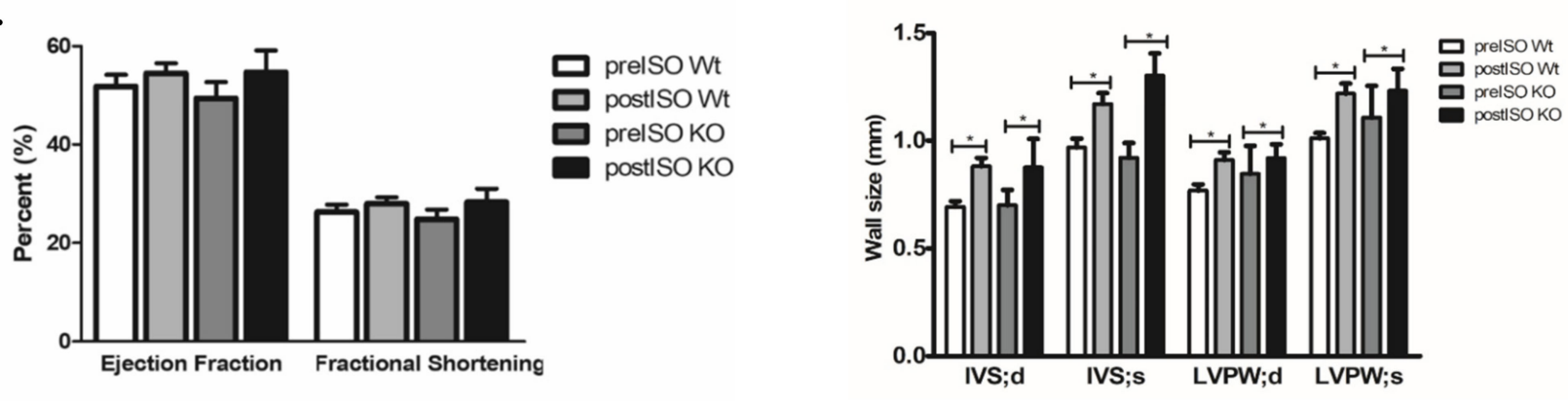

Figure 7. Echocardiography and left ventricular function in ISO challenged Wt and SLMAP3-KO hearts. (A) Representative short-axis m-mode images of Wt and $\mathrm{KO}$ mice preISO and postISO. (B) Left ventricular functional analysis and changes in wall dimensions between pre-/postISO treated $\mathrm{Wt}$ and $\mathrm{KO}$ hearts. $n=7 .{ }^{*} \rho<0.05, \rho$ value was calculated using a paired two-way $t$-test. 


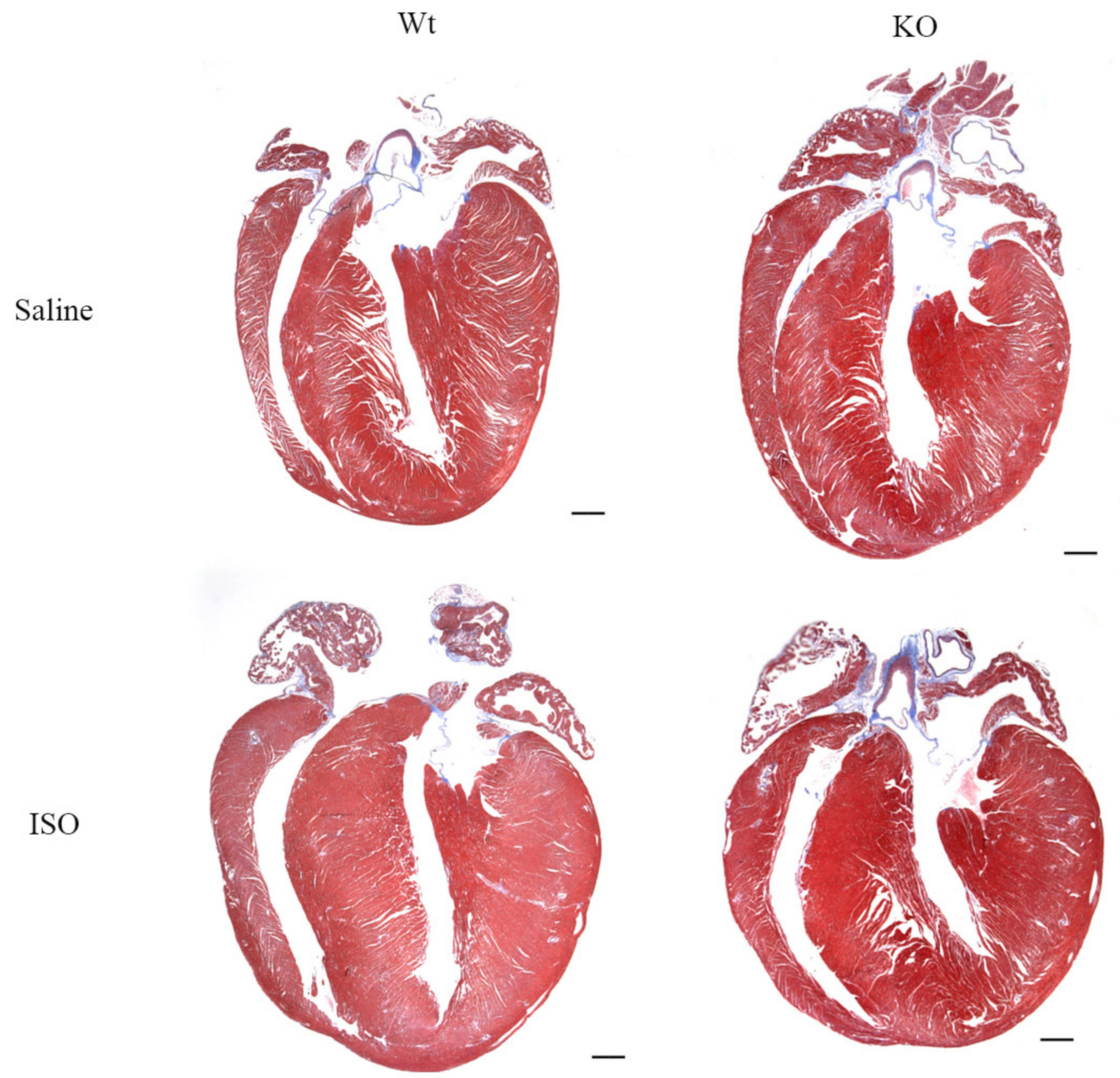

Figure 8. Histological analysis of ISO challenged Wt and SLMAP3-KO mouse hearts. Representative sectioning of Wt or $\mathrm{KO}$ hearts after one week of saline or ISO with Masson's trichome stain to visualize the muscle and fibrosis. Scale bar = $500 \mu \mathrm{m} \times 12.5$ magnification.

\subsection{STRIPAK Complex and Hippo Signaling in SLMAP3-KO Myocardium}

Several studies have indicated that SLMAP3, via its FHA domain, can regulate Hippo signaling by recruiting MST1/MST2, which comprises STRIPAK. The core STRIPAK components (striatin, PP2A-A, PP2A-C) were assessed by western blotting of cardiac lysates together with MST1 $/ 2$ and YAP. Striatin $(n=6, \rho=0.58)$, PP2A-A $(n=6, \rho=0.69)$ and PP2A-C $(n=6, \rho=0.28)$ protein levels were not significantly altered in adult SLMAP3-KO hearts compared to Wt (Figure 9). The phosphorylation of MST1/2 (T183) and YAP (S127) was evaluated and quantified, and the phospho-YAP to total YAP ratio $(n=6, \rho=0.34)$ and phospho-MST1 to total MST1 $(n=6, \rho=0.36)$ was not significantly altered in postnatal SLMAP3-KO hearts compared to Wt (Figure 9). 

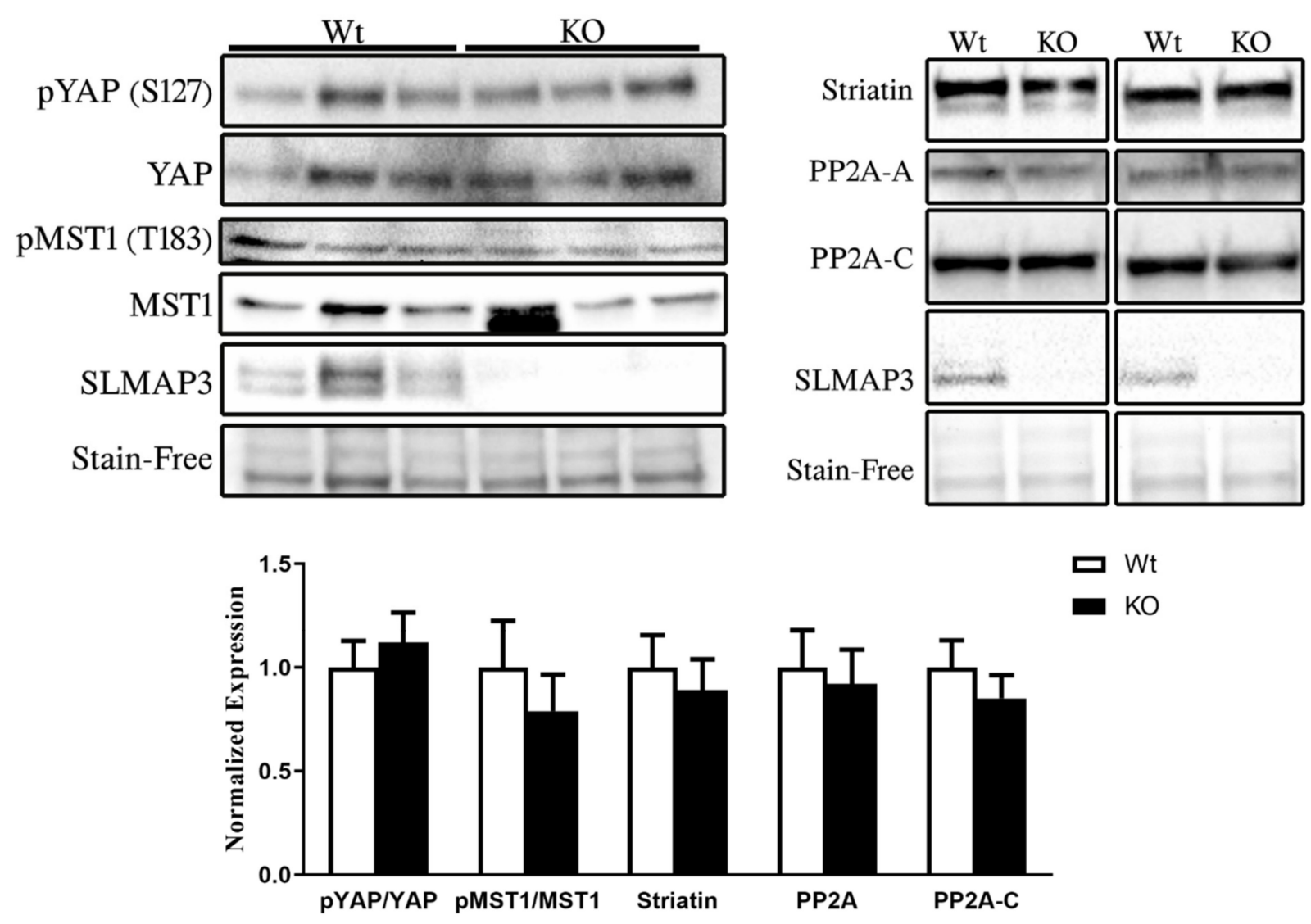

Figure 9. STRIPAK complex and Hippo signaling in SLMAP3-KO hearts. Western blot of Wt and KO adult mouse heart lysates evaluating protein expression STRIPAK components (striatin, PP2A-A and PP2A-C) and phosphorylation of MST1 (T183) and YAP (S127). Bar graphs represent the expression of indicated proteins by normalizing with total protein visualized by Stain-Free as a loading control or to total MST1 or total YAP. $n=6$.

\subsection{Nav1.5 Expression and Electrophysiology in SLMAP3-Deficient Myocardium}

Mutations in the human SLMAP gene have been linked to Brugada with inappropriate Nav1.5 shuttling [24,26]. Western blot analysis indicated that there was a modest, albeit significant, decrease of Nav1.5 protein levels in $\mathrm{KD}(-9.81 \% \pm-6.00 \%, \rho<0.05, n=3)$ and KO heart lysates $(-16.66 \% \pm-6.69 \%, \rho<0.05, n=3)$ when compared to Wt (Figure 10A). Since Nav1.5 plays a major role in initiating the E-C coupling cascade, we determined if a modest decrease of Nav1.5 can impact the electrophysiology of SLMAP3-deficient hearts [41]. A 6-lead surface electrocardiography (ECG) in mice aged up to 28 weeks showed no significant changes between Wt and SLMAP3-deficient hearts (Figure 10B). Electrical properties were quantified, and $\mathrm{P}, \mathrm{R}, \mathrm{Q}, \mathrm{S}$ and $\mathrm{T}$ waves in each ECG reading are defined in Figure 10B. Our analysis showed that the PP duration, PR segment, PR interval, QRS duration and QTc interval were not significantly altered between Wt and SLMAP3-deficient hearts (Table 2). Further, P, R and $\mathrm{S}$ amplitudes did not significantly vary in SLMAP3-deficient hearts when compared to $\mathrm{Wt}$ (Table 2). Therefore, the decrease in Nav1.5 protein expression did not significantly alter cardiac electrophysiology in SLMAP3-deficient hearts. 
A.
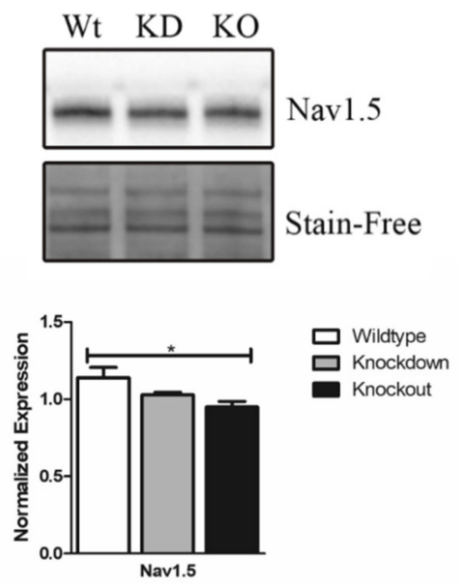

B.

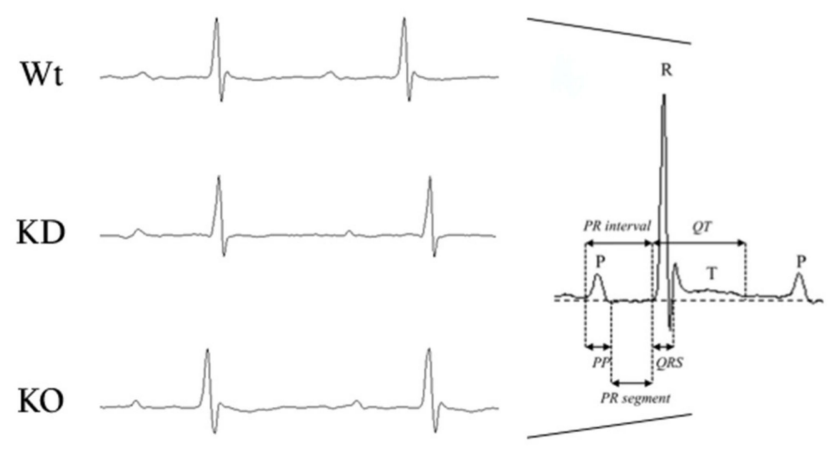

Figure 10. Nav1.5 expression and electrocardiography in SLMAP3-deficient hearts. (A) Western blot with anti-Nav1.5 in $\mathrm{Wt}, \mathrm{KD}$ and KO adult mouse hearts lysates. Bar graphs represent quantification of Nav1.5 expression normalized to total protein loading visualized by Stain-Free. (B) Representative electrograms of lead ii acquired by surface 6-lead ECG in Wt, KD and KO adult mice. $n=3 .{ }^{*} \rho<0.05, \rho$ value was calculated using ANOVA statistical analysis.

Table 2. Electrocardiography of SLMAP3-deficient hearts. Parameters of electrocardiography in Wt, KD and KO adult mice (detailed in materials and methods). $n=3$. $\rho$ value was calculated using ANOVA statistical analysis.

\begin{tabular}{ccccc}
\hline $\begin{array}{c}\text { Electrocardiography } \\
\text { Parameter }\end{array}$ & Wt & KD & KO & $\rho$-Value \\
\hline RR (ms) & $123.370 \pm 3.163$ & $127.5 \pm 2.644$ & $123.407 \pm 3.271$ & 0.5738 \\
HR (bpm) & $487.4 \pm 12.771$ & $471.224 \pm 9.847$ & $487.248 \pm 13.116$ & 0.5787 \\
PP duration (ms) & $8.941 \pm 0.177$ & $8.168 \pm 0.737$ & $8.023 \pm 0.651$ & 0.5197 \\
PR segment (ms) & $29.738 \pm 0.684$ & $29.429 \pm 4.568$ & $28.781 \pm 1.557$ & 0.9148 \\
PR interval (ms) & $45.185 \pm 0.737$ & $44.685 \pm 2.428$ & $41.558 \pm 0.883$ & 0.2764 \\
QRS duration (ms) & $11.954 \pm 1.289$ & $10.298 \pm 0.549$ & $11.613 \pm 0.773$ & 0.4568 \\
QTc interval (ms) & $46.010 \pm 2.508$ & $40.969 \pm 6.033$ & $45.713 \pm 2.166$ & 0.6262 \\
P amplitude (mV) & $0.0531 \pm 0.009$ & $0.069 \pm 0.01$ & $0.067 \pm 0.003$ & 0.3765 \\
R amplitude (mV) & $25.670 \pm 0.730$ & $21.351 \pm 0.789$ & $0.620 \pm 0.027$ & 0.5823 \\
S amplitude (mV) & $-0.248 \pm 0.023$ & $-0.204 \pm 0.031$ & $-0.219 \pm 0.046$ & 0.6749 \\
\hline
\end{tabular}

\section{Discussion}

Mutations in SLMAP have been linked to Brugada, while it has also been shown to be a component of the STRIPAK complex involved in regulating Hippo signaling. To define the precise role of SLMAP within postnatal myocardium, we generated a cardiac-specific knockout of SLMAP using the cre-lox system by targeting exon 3 of the SLMAP gene with a view to nullify its expression. We employed the $\alpha$ MHC-MerCreMer method to define the impact of SLMAP loss on myocardium in a temporal manner, i.e., in the postnatal myocardium by using tamoxifen to activate cre and excise floxed SLMAP [33].

To our surprise, cleaving exon 3 of the mouse SLMAP gene only nullified the expression of SLMAP3. The transcript and protein levels of SLMAP1 and SLMAP2 isoforms remained unchanged, while SLMAP3 levels were significantly decreased in KD and lost in KO hearts. We hypothesized that the loss of SLMAP3 would impact the growth/function of postnatal myocardium since SLMAP3 houses the FHA domain, which was shown to integrate MST1/2 to regulate Hippo signaling [18-21]. In addition, the loss of SLMAP3 may influence Nav 1.5 shuttling and the electrical properties of the myocardium [24]. ECHO analysis indicated that SLMAP3-deficient hearts showed no significant changes in cardiac structure or function in the acute or chronic loss of SLMAP3. Administration of ISO to experimental animals did not expose any underlying phenotypes to this stressor of the myocardium. ECHO and histological analysis indicated an identical hypertrophic phenotype in iso-treated $\mathrm{KO}$ and Wt hearts implying that SLMAP3 loss had no effect on 
the stress response. These results suggest that SLMAP3 does not play a role in this aspect of cardiac structure or function, and this may be due to compensation by the abundant levels of SLMAP1 and SLMAP2 that remain unchanged in the SLMAP3-KO myocardium. SLMAP1 and SLMAP2 could compensate due to the significant identity between these isoforms and their robust expression in the myocardium relative to SLMAP3 [5]. We theorize that expression of SLMAP1 and SLMAP2 is not affected by the cleavage of exon 3 because they may be regulated by alternate promoters [42]. SLMAP1 and SLMAP2 are truncated variants of SLMAP3 and contain unique in-frame start codons at exon 10 and 16, and expression may be driven by promoters upstream of these start codons [2].

Several studies have indicated that the expression and localization of Nav1.5 can be impacted by SLMAP3 [24,26]. Ishikawa et al. linked mutations in the human SLMAP gene at exon 8 (SLMAP3-specific) and exon 21 (all SLMAP isoforms) in Brugada patients. When mutated SLMAP3 or SLMAP1 proteins were expressed in HEK293, they inhibited the trafficking of expressed hNav1.5 to the cell surface [24]. In SLMAP3-KO hearts, there was a minor but significant decrease in Nav1.5 protein expression. However, ECG analysis of adult mice with cardiac SLMAP3-deficiency displayed no significant differences in any parameters when compared to Wt. Although Nav1.5 trafficking was not assessed, the modest decrease in Nav1.5 and lack of changes in electrophysiology indicates that the loss of SLMAP3 has no impact in this respect. SLMAP1 and SLMAP2 levels remain unchanged and potentially could compensate for SLMAP3 loss of function. However, we have shown that the SLMAP3 transgenic myocardium had decreased transcript levels of Nav1.5 and Serca2a, but this was not seen in SLMAP1 transgenic hearts. Thus SLMAP3 may uniquely modulate gene expression in vivo, further implying a relationship between SLMAP3 and Nav1.5 [26]. SLMAP isoforms can homo- and heterodimerize via coiled-coil domains [5], and the overexpression of SLMAP3 could potentially sequester endogenous SLMAPs and interacting proteins to subcellular domains, impacting the mechanism that regulates expression/trafficking of Nav1.5, although this needs to be fully interrogated. Researchers have shown that mice with $\sim 50 \%$ decrease of Nav1.5 expression display defects in atrioventricular conduction parameters without presenting any obvious cardiac phenotypes [43]. This was also noted in our SLMAP3 overexpressing transgenic mice as Nav1.5 transcript levels were reduced by 55\%, displaying atrioventricular conduction defects with no overt defects in the myocardium [26].

The fork head-associated (FHA) domain in SLMAP3 has been shown to recruit MST1/2 kinase to negatively regulate the Hippo pathway via the STRIPAK complex [44,45]. Evidence indicates that loss of SLMAP3 in human cells and Drosophila positively regulates Hippo signaling i.e., increased phosphorylation of MST1/2 (T183) and YAP (S127) [18-21,46]; however, no significant differences were noted in phosphorylation of MST1/2 or YAP in SLMAP3-null hearts compared to wildtype myocardium. When Hippo signaling is upregulated in adult mouse hearts via overexpression of cardiac-specific MST1/2 and/or deletion of YAP, it leads to cardiomyopathies and heart failure $[47,48]$. Therefore, if the loss of SLMAP3 would have affected Hippo signaling similarly, it should result in similar phenotypes in postnatal mouse hearts. It is notable that Hippo deficiency in adult myocardium leads to dysfunction in pressure overload [49] while it reversed the systolic heart failure after infarction [50]. Although we used ISO to stress the heart, we did not observe any cardiac dysfunction or changes in Hippo signaling. Whether injury, such as myocardial infarction or pressure overload, would impact Hippo in SLMAP3 deficient hearts, and function remains to be investigated [51]. If and how the abundant expression of SLMAP1 and SLMAP2 isoforms in postnatal myocardium can compensate for SLMAP3 loss in terms of Hippo signaling and function remains to be examined. The lack of change in Hippo signaling due to SLMAP3 loss could also be due to the extremely low proliferative rates of cardiomyocytes in postnatal heart development since Hippo signaling reaches its peak as early as 10-days after birth [52,53]. Thus, the lack of phenotype in SLMAP3-deficient postnatal myocardium could be due to a lack of proliferative programming in matured cardiomyocytes [54,55]. Studies to evaluate the impact of SLMAP3 by targeting its 
prenatal/perinatal expression, where cardiomyocyte proliferation/growth is critical, are now in progress together with nullifying SLMAP1 and SLMAP2 expression [56,57].

\section{Conclusions}

In conclusion, cardiac-specific loss of SLMAP3 in postnatal myocardium did not significantly alter the structure/function or activity of Hippo signaling in mouse hearts. Although a minor decrease in Nav1.5 expression was noted, no effect on cardiac electrophysiology was evident in adult SLMAP3-deficient hearts. We theorize that isoforms SLMAP1 and SLMAP2 may compensate for SLMAP3 due to their abundant expression in the adult myocardium. To further investigate in vivo roles of SLMAPs, studies are in progress to nullify the expression of all SLMAP isoforms during prenatal/perinatal cardiac development.

Supplementary Materials: The following are available online at https://www.mdpi.com/article/ 10.3390/cardiogenetics11040018/s1, Table S1: ECHO analysis on young and old SLMAP3-deficient hearts. Left ventricular functional analysis, measurements of wall sizes and intradiameter during diastole and systole in 5-week and 24-week old $\mathrm{Wt}, \mathrm{KD}$, and $\mathrm{KO}$ mice. $\rho$-value was calculated using ANOVA statistics analysis. $n=6$. IVS; intraventricular septum, LVID; Left ventricular intradiameter, LVPW; Left ventricular posterior wall, EF; ejection fraction, FS; fractional shortening, LV mass; left ventricular mass, $\mathrm{LV}$ volume; left ventricular volume, $\mathrm{d} / \mathrm{s}$; diastole/systole, $\mathrm{LV} / \mathrm{BW}$; left ventricular mass/body weight.; Table S2: ECHO analysis on ISO-treated SLMAP3-KO hearts. Measurement of left ventricular mass and wall sizes during diastole and systole in $\mathrm{Wt}$ and $\mathrm{KO}$ mouse hearts before and after treatment with ISO. $\rho$-value was calculated using two-tailed student. $n=7$. IVS; intraventricular septum, LVID; Left ventricular intradiameter, LVPW; Left ventricular posterior wall, EF; ejection fraction, FS; fractional shortening, LV mass; left ventricular mass, LV volume; left ventricular volume, $\mathrm{d} / \mathrm{s}$; diastole/systole.

Author Contributions: Conceptualization, T.R., M.S., J.B. and B.S.T.; methodology, T.R., M.S., J.B. and B.S.T.; data analysis, T.R., J.M. and B.S.T.; investigation, T.R., J.M., M.S. and J.B.; visualization: T.R. and B.S.T.; writing — original draft preparation, T.R.; Project administration: M.S. and B.S.T.; writing—review and editing, B.S.T.; supervision M.S. and B.S.T.; funding acquisition, B.S.T. All authors have read and agreed to the published version of the manuscript.

Funding: This research was funded by the Canadian Institute of Health Research project grant, grant number 220996-151999, to B.S.T.

Institutional Review Board Statement: Mice were handled in accordance with the guidelines set by Canadian Council on Animal Care, Guide to the Care and Use of Experimental Animals, 2 vols. (Ottawa, Ont.: CCAC, 1980-1993) and Animals for Research Act, R.S.O. 1990, c.A. 22. All animal protocols and procedures were approved by the Animal Care Committee of the University of Ottawa (Protocol \#: CMM-1725 and CMM-1723, Renewed: December 2020).

Informed Consent Statement: Not applicable.

Data Availability Statement: The data presented in this study are available on request from the corresponding author.

Acknowledgments: We would like to thank Mona Nemer and her lab staff for providing the $\alpha \mathrm{MHC}$ MerCreMer animals, sharing breeding strategies and Rick Seymour for assisting our ECHO analysis.

Conflicts of Interest: The authors declare that the research was conducted in the absence of any commercial or financial relationships that could be construed as a potential conflict of interest.

\section{References}

1. Wigle, J.; Demchyshyn, L.; Pratt, M.; Staines, W.; Salih, M.; Tuana, B. Molecular cloning, expression, and chromosomal assignment of sarcolemmal-associated proteins. A family of acidic amphipathic alpha-helical proteins associated with the membrane. J. Biol. Chem. 1997, 272, 32384-32394. [CrossRef]

2. Wielowieyski, P.; Sevinc, S.; Guzzo, R.; Salih, M.; Wigle, J.; Tuana, B. Alternative splicing, expression, and genomic structure of the 3' region of the gene encoding the sarcolemmal-associated proteins (SLAPs) defines a novel class of coiled-coil tail-anchored membrane proteins. J. Biol. Chem. 2000, 275, 38474-38481. [CrossRef] 
3. Guzzo, R.; Wigle, J.; Salih, M.; Moore, E.; Tuana, B. Regulated expression and temporal induction of the tail-anchored sarcolemmalmembrane-associated protein is critical for myoblast fusion. Biochem. J. 2004, 381, 599-608. [CrossRef]

4. Byers, J.; Guzzo, R.; Salih, M.; Tuana, B. Hydrophobic profiles of the tail anchors in SLMAP dictate subcellular targeting. BMC Cell Biol. 2009, 10, 48. [CrossRef] [PubMed]

5. Guzzo, R.; Sevinc, S.; Salih, M.; Tuana, B. A novel isoform of sarcolemmal membrane-associated protein (SLMAP) is a component of the microtubule organizing center. J. Cell Sci. 2004, 117 Pt 11, 2271-2281. [CrossRef] [PubMed]

6. Letunic, I.; Bork, P. 20 years of the SMART protein domain annotation resource. Nucleic Acids Res. 2018, 46, D493-D496. [CrossRef] [PubMed]

7. Mahajan, A.; Yuan, C.; Lee, H.; Chen, E.; Wu, P.; Tsai, M. Structure and function of the phosphothreonine-specific FHA domain. Sci. Signal. 2008, 1, re12. [CrossRef] [PubMed]

8. Couzens, A.; Knight, J.; Kean, M.; Teo, G.; Weiss, A.; Dunham, W.; Lin, Z.; Bagshaw, R.; Sicheri, F.; Pawson, T.; et al. Protein interaction network of the mammalian Hippo pathway reveals mechanisms of kinase-phosphatase interactions. Sci. Signal. 2013, 6, rs15. [CrossRef] [PubMed]

9. Goudreault, M.; D'Ambrosio, L.; Kean, M.; Mullin, M.; Larsen, B.; Sanchez, A.; Chaudhry, S.; Chen, G.; Sicheri, F.; Nesvizhskii, A.; et al. A PP2A Phosphatase High Density Interaction Network Identifies a Novel Striatin-interacting Phosphatase and Kinase Complex Linked to the Cerebral Cavernous Malformation 3 (CCM3) Protein. Mol. Cell Proteom. 2009, 8, 157-171. [CrossRef]

10. Zheng, Y.; Pan, D. The Hippo Signaling Pathway in Development and Disease. Dev. Cell 2019, 50, 264-282. [CrossRef]

11. Wang, J.; Liu, S.; Heallen, T.; Martin, J. The Hippo pathway in the heart: Pivotal roles in development, disease, and regeneration. Nat. Rev. Cardiol. 2018, 15, 672-684. [CrossRef] [PubMed]

12. Misra, J.; Irvine, K. The Hippo Signaling Network and Its Biological Functions. Annu. Rev. Genet. 2018, 52, 65-87. [CrossRef] [PubMed]

13. Zhou, Q.; Li, L.; Zhao, B.; Guan, K.L. The Hippo pathway in heart development, regeneration, and diseases. Circ. Res. 2015, 116, 1431-1447. [CrossRef] [PubMed]

14. Von Gise, A.; Lin, Z.; Schlegelmilch, K.; Honor, L.; Pan, G.; Buck, J.; Ma, Q.; Ishiwata, T.; Zhou, B.; Camargo, F.; et al. YAP1, the nuclear target of Hippo signaling, stimulates heart growth through cardiomyocyte proliferation but not hypertrophy. Proc. Natl. Acad. Sci. USA 2012, 109, 2394-2399. [CrossRef]

15. Xin, M.; Kim, Y.; Sutherland, L.; Murakami, M.; Qi, X.; McAnally, J.; Porrello, E.; Mahmoud, A.; Tan, W.; Shelton, J.; et al. Hippo pathway effector Yap promotes cardiac regeneration. Proc. Natl. Acad. Sci. USA 2013, 110, 13839-13844. [CrossRef]

16. Heallen, T.; Zhang, M.; Wang, J.; Bonilla-Claudio, M.; Klysik, E.; Johnson, R.; Martin, J. Hippo pathway inhibits Wnt signaling to restrain cardiomyocyte proliferation and heart size. Science 2011, 332, 458-461. [CrossRef] [PubMed]

17. Byun, J.; Del Re, D.; Zhai, P.; Ikeda, S.; Shirakabe, A.; Mizushima, W.; Miyamoto, S.; Brown, J.; Sadoshima, J. Yes-associated protein (YAP) mediates adaptive cardiac hypertrophy in response to pressure overload. J. Biol. Chem. 2019, 294, 3603-3617. [CrossRef]

18. Jeong, B.; Bae, S.; Ni, L.; Zhang, X.; Bai, X.; Luo, X. Cryo-EM structure of the Hippo signaling integrator human STRIPAK. Nat. Struct. Mol. Biol. 2021, 28, 290-299. [CrossRef]

19. Hauri, S.; Wepf, A.; van Drogen, A.; Varjosalo, M.; Tapon, N.; Aebersold, R.; Gstaiger, M. Interaction proteome of human Hippo signaling: Modular control of the co-activator YAP1. Mol. Syst. Biol. 2013, 9, 713. [CrossRef]

20. Bae, S.; Ni, L.; Osinski, A.; Tomchick, D.; Brautigam, C.; Luo, X. SAV1 promotes Hippo kinase activation through antagonizing the PP2A phosphatase STRIPAK. eLife 2017, 6, e30278. [CrossRef]

21. Zheng, Y.; Liu, B.; Wang, L.; Lei, H.; Pulgar Prieto, K.; Pan, D. Homeostatic Control of Hpo/MST Kinase Activity through Autophosphorylation-Dependent Recruitment of the STRIPAK PP2A Phosphatase Complex. Cell Rep. 2017, 21, 3612-3623. [CrossRef]

22. Guzzo, R.M.; Salih, M.; Moore, E.D.; Tuana, B. Molecular properties of cardiac tail-anchored membrane protein SLMAP are consistent with structural role in arrangement of excitation-contraction coupling apparatus. Am. J. Physiol. Heart Circ. Physiol. 2005, 288, H1810-H1819. [CrossRef] [PubMed]

23. Nader, M.; Westendorp, B.; Hawari, O.; Salih, M.; Stewart, A.F.; Leenen, F.H.; Tuana, B.S. Tail-anchored membrane protein SLMAP is a novel regulator of cardiac function at the sarcoplasmic reticulum. Am. J. Physiol. Heart Circ. Physiol. 2012, 302, 1138-1145. [CrossRef]

24. Ishikawa, T.; Sato, A.; Marcou, C.; Tester, D.; Ackerman, M.; Crotti, L.; Schwartz, P.; On, Y.; Park, J.; Nakamura, K.; et al. A novel disease gene for Brugada syndrome: Sarcolemmal membrane-associated protein gene mutations impair intracellular trafficking of hNav1.5. Circ. Arrhythm Electrophysiol. 2012, 5, 1098-1107. [CrossRef]

25. Brugada, R.; Campuzano, O.; Sarquella-Brugada, G.; Brugada, J.; Brugada, P. Brugada syndrome. Methodist Debakey Cardiovasc. J. 2014, 10, 25-28. [CrossRef]

26. Mlynarova, J.; Trentin-Sonoda, M.; Gaisler da Silva, F.; Major, J.; Salih, M.; Carneiro-Ramos, M.; Tuana, B. SLMAP3 isoform modulates cardiac gene expression and function. PLOS ONE 2019, 14, e0214669. [CrossRef]

27. Bustin, S.A. Quantification of mRNA using real-time reverse transcription PCR (RT-PCR): Trends and problems. J. Mol. Endocrinol. 2002, 29, 23-29. [CrossRef] [PubMed]

28. Mitchell, G.; Jeron, A.; Koren, G. Measurement of heart rate and Q-T interval in the conscious mouse. Am. J. Physiol. 1998, 274, H747-H751. [CrossRef] [PubMed] 
29. Aken, B.; Ayling, S.; Barrell, D.; Clarke, L.; Curwen, V.; Fairley, S.; Fernandez, B.J.; Billis, K.; García, G.C.; Hourlier, T.; et al. The Ensembl gene annotation system. Database 2016, 2016, baw093. [CrossRef] [PubMed]

30. Consortium, U. UniProt: A worldwide hub of protein knowledge. Nucleic Acids Res. 2019, 47, D506-D515. [CrossRef]

31. Schmidt, T.; Samaras, P.; Frejno, M.; Gessulat, S.; Barnert, M.; Kienegger, H.; Krcmar, H.; Schlegl, J.; Ehrlich, H.C.; Aiche, S.; et al. ProteomicsDB. Nucleic Acids Res. 2018, 4, D1271-D1281. [CrossRef]

32. Davis, J.; Maillet, M.; Miano, J.; Molkentin, J. Lost in transgenesis: A user's guide for genetically manipulating the mouse in cardiac research. Circ. Res. 2012, 111, 761-777. [CrossRef] [PubMed]

33. Sohal, D.S.; Nghiem, M.; Crackower, M.A.; Witt, S.A.; Kimball, T.R.; Tymitz, K.M.; Penninger, J.M.; Molkentin, J.D. Temporally regulated and tissue-specific gene manipulations in the adult and embryonic heart using a tamoxifen-inducible Cre protein. Circ. Res. 2001, 89, 20-25. [CrossRef]

34. Demayo, J.; Wang, J.; Liang, D.; Zhang, R.; Demayo, F. Genetically Engineered Mice by Pronuclear DNA microinjection. Curr. Protoc. Mouse Biol. 2012, 2, 245-262. [CrossRef] [PubMed]

35. Ayadi, A.; Ferrand, G.; Cruz, I.; Warot, X. Mouse Breeding and Colony Management. Curr. Protoc. Mouse Biol. $2011,1,239-264$.

36. Kuhn, R.; Torres, R.M. Cre/LoxP Recombination System and Gene Targeting. Transgenesis Tech. 2002, 180, 175-204.

37. Bersell, K.; Choudhury, S.; Mollova, M.; Polizzotti, B.D.; Ganapathy, B.; Walsh, S.; Wadugu, B.; Arab, S.; Kühn, B. Moderate and high amounts of tamoxifen in $\alpha \mathrm{MHC}$-MerCreMer mice induce a DNA damage response, leading to heart failure and death. Dis. Models Mech. 2013, 6, 1459-1469.

38. Yang, X.; Liu, Y.; Rhaleb, N.; Kurihara, N.; Kim, H.; Carretero, O. Echocardiographic assessment of cardiac function in conscious and anesthetized mice. Am. J. Physiol. 1999, 277, 1967-1974. [CrossRef]

39. Zhang, G.; Kimura, S.; Nishiyama, A.; Shokoji, T.; Rahman, M.; Yao, L.; Nagai, Y.; Fujisawa, Y.; Miyatake, A.; Abe, Y. Cardiac oxidative stress in acute and chronic isoproterenol-infused rats. Cardiovasc. Res. 2005, 65, 230-238. [CrossRef] [PubMed]

40. Major, J.; Salih, M.; Tuana, B. Interplay between the E2F pathway and $\beta$-adrenergic signaling in the pathological hypertrophic response of myocardium. J. Mol. Cell Cardiol. 2015, 84, 179-190. [CrossRef]

41. Dong, C.; Wang, Y.; Ma, A.; Wang, T. Life Cycle of the Cardiac Voltage-Gated Sodium Channel NaV1.5. Front. Physiol. 2020, 11, 609733. [CrossRef]

42. Ayoubi, T.; Van De Ven, W. Regulation of gene expression by alternative promoters. FASEB J. 1996, 4, 453-460. [CrossRef]

43. Papadatos, G.; Wallerstein, P.; Head, C.; Ratcliff, R.; Brady, P.; Benndorf, K.; Saumarez, R.; Trezise, A.; Huang, C.; Vandenberg, J.; et al. Slowed conduction and ventricular tachycardia after targeted disruption of the cardiac sodium channel gene Scn5a. Proc. Natl. Acad. Sci. USA 2002, 99, 6210-6215. [CrossRef]

44. Bae, S.; Ni, L.; Luo, X. STK25 suppresses Hippo signaling by regulating SAV1-STRIPAK antagonism. eLife 2020, 9, e54863. [CrossRef]

45. Chen, R.; Xie, R.; Meng, Z.; Ma, S.; Guan, K. STRIPAK integrates upstream signals to initiate the Hippo kinase cascade. Nat. Cell Biol. 2019, 21, 1565-1577. [CrossRef]

46. Kaya-Çopur, A.; Marchiano, F.; Hein, M.; Alpern, D.; Russeil, J.; Luis, N.; Mann, M.; Deplancke, B.; Habermann, B.; Schnorrer, F. The Hippo pathway controls myofibril assembly and muscle fiber growth by regulating sarcomeric gene expression. Elife 2021, 10, e63726. [CrossRef] [PubMed]

47. Del Re, D.; Yang, Y.; Nakano, N.; Cho, J.; Zhai, P.; Yamamoto, T.; Zhang, N.; Yabuta, N.; Nojima, H.; Pan, D.; et al. Yes-associated protein isoform 1 (Yap1) promotes cardiomyocyte survival and growth to protect against myocardial ischemic injury. J. Biol. Chem. 2013, 288, 3977-3988. [CrossRef] [PubMed]

48. Yamamoto, S.; Yang, G.; Zablocki, D.; Liu, J.; Hong, C.; Kim, S.; Soler, S.; Odashima, M.; Thaisz, J.; Yehia, G.; et al. Activation of Mst1 causes dilated cardiomyopathy by stimulating apoptosis without compensatory ventricular myocyte hypertrophy. J. Clin. Invest. 2003, 111, 1463-1474. [CrossRef] [PubMed]

49. Ikeda, S.; Mizushima, W.; Sciarretta, S.; Abdellatif, M.; Zhai, P.; Mukai, R.; Fefelova, N.; Oka, S.; Nakamura, M.; Del Re, D.; et al. Hippo Deficiency Leads to Cardiac Dysfunction Accompanied by Cardiomyocyte Dedifferentiation During Pressure Overload. Circ. Res. 2019, 124, 292-305. [CrossRef] [PubMed]

50. Leach, J.; Heallen, T.; Zhang, M.; Rahmani, M.; Morikawa, Y.; Hill, M.; Segura, A.; Willerson, J.; Martin, J. Hippo pathway deficiency reverses systolic heart failure after infarction. Nature 2017, 550, 260-264. [CrossRef]

51. Wang, P.; Mao, B.; Luo, W.; Wei, B.; Jiang, W.; Liu, D.; Song, L.; Ji, G.; Yang, Z.; Lai, Y.; et al. The alteration of Hippo/YAP signaling in the development of hypertrophic cardiomyopathy. Basic Res. Cardiol. 2014, 109, 435-438. [CrossRef]

52. Heallen, T.; Morikawa, Y.; Leach, J.; Tao, G.; Willerson, J.T.; Johnson, R.L.; Martin, J.F. Hippo signaling impedes adult heart regeneration. Development 2013, 140, 4683-4690. [CrossRef] [PubMed]

53. Bergmann, O.; Bhardwaj, R.; Bernard, S.; Zdunek, S.; Barnabé-Heider, F.; Walsh, S.; Zupicich, J.; Alkass, K.; Buchholz, B.; Druid, H.; et al. Evidence for cardiomyocyte renewal in humans. Science 2009, 324, 98-102. [CrossRef] [PubMed]

54. Alkass, K.; Panula, J.; Westman, M.; Wu, T.; Guerquin-Kern, J.; Bergmann, O. No Evidence for Cardiomyocyte Number Expansion in Preadolescent Mice. Cell 2015, 163, 1026-1036. [CrossRef]

55. Heallen, T.; Kadow, Z.; Wang, J.; Martin, J. Determinants of Cardiac Growth and Size. Cold Spring Harb. Perspect. Biol. 2020, 2, a037150. [CrossRef] [PubMed] 
56. Yutzey, K. Cardiomyocyte Proliferation: Teaching an Old Dogma New Tricks. Circ. Res. 2017, 120, 627-629. [CrossRef]

57. Porrello, E.; Mahmoud, A.; Simpson, E.; Hill, J.; Richardson, J.; Olson, E.; Sadek, H. Transient regenerative potential of the neonatal mouse heart. Science 2011, 331, 1078-1080. [CrossRef] 